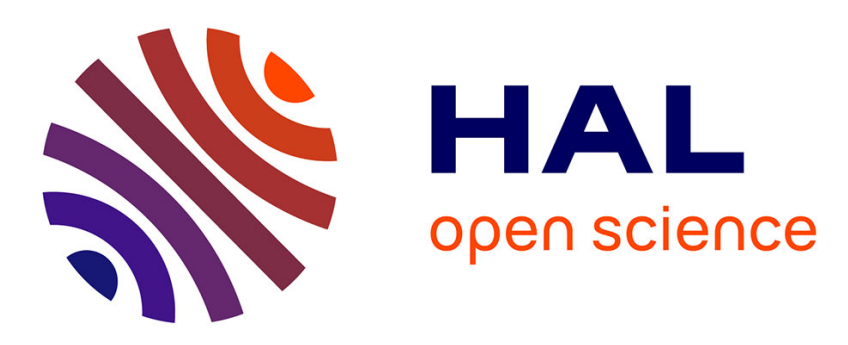

\title{
Controlled monohalogenation of phosphonates: A new route to pure $\alpha$-monohalogenated diethyl benzylphosphonates
}

Bogdan Iorga, Frédéric Eymery, Philippe Savignac

\section{- To cite this version:}

Bogdan Iorga, Frédéric Eymery, Philippe Savignac. Controlled monohalogenation of phosphonates: A new route to pure $\alpha$-monohalogenated diethyl benzylphosphonates. Tetrahedron, 1999, 55 (9), pp.2671-2686. 10.1016/s0040-4020(99)00037-x . hal-03161386

\author{
HAL Id: hal-03161386 \\ https://hal.science/hal-03161386
}

Submitted on 10 Mar 2021

HAL is a multi-disciplinary open access archive for the deposit and dissemination of scientific research documents, whether they are published or not. The documents may come from teaching and research institutions in France or abroad, or from public or private research centers.
L'archive ouverte pluridisciplinaire $\mathbf{H A L}$, est destinée au dépôt et à la diffusion de documents scientifiques de niveau recherche, publiés ou non, émanant des établissements d'enseignement et de recherche français ou étrangers, des laboratoires publics ou privés. 


\title{
Controlled Monohalogenation of Phosphonates: A New Route to Pure $\alpha$-Monohalogenated Diethyl Benzylphosphonates
}

\author{
Bogdan Iorga, Frédéric Eymery and Philippe Savignac \\ Laboratoire Hétéroatomes et Coordination, UMR CNRS 7653, DCPH, Ecole Polytechnique, \\ 91128 Palaiseau Cedex, France*
}

\begin{abstract}
Starting from diethyl benzylphosphonates, a wide variety of diethyl $\alpha$-monofluoro, chloro, bromo and iodobenzylphosphonates have been obtained in pure form by a one-pot procedure. This high yielding method implies the intermediate protection of the benzyl anion with TMSCl followed by halogenation with an electrophilic halogenating reagent.
\end{abstract}

\section{INTRODUCTION}

The interest in generating dialkyl $\alpha$-monohalogenobenzylphosphonates lies for one part in their potential for the synthesis of $\alpha$-halogenoalkenes and alkynes which are important intermediates in organic synthesis and material science. ${ }^{1-4}$ On the other hand, substitution of a hydrogen atom in $\alpha-\mathrm{CH}_{2}$ position by fluorine in enzyme substrate phosphonic analogues is widely practised in various areas of bio-organic and medicinal chemistry with the aim to prepare better mimics of phosphate parents. ${ }^{5}$ However, a practical, general and high yielding method for the synthesis of pure dialkyl $\alpha$-monohalogenobenzylphosphonates has not yet been described. A number of approaches for the introduction of an halogen at the $\alpha-\mathrm{CH}_{2}$ position of phosphonates has been reported and in order to limit the scope of this introduction only the four main processes are discussed below :

(a) The most widely used synthetic method is the nucleophilic halogenation of diethyl $\alpha$ hydroxybenzylphosphonates, obtained via the Pudovik reaction between diethyl phosphite and aromatic aldehydes, with a large variety of halogenating reagents: $\mathrm{CHFCl}_{-} \mathrm{CF}_{2} \mathrm{NEt}_{2}{ }^{6}$ and $\mathrm{Et}_{2} \mathrm{~N}-\mathrm{SF}_{3}$ (DAST) ${ }^{7}$ for fluorination, $\mathrm{POCl}_{3},{ }^{1-3} \mathrm{PPh}_{3} / \mathrm{CCl}_{4},{ }^{8} \mathrm{SOCl}_{2}{ }^{9}$ for chlorination, $\mathrm{PPh}_{3} / \mathrm{CBr}_{4},{ }^{10} \mathrm{PPh}_{3} \mathrm{Br}_{2} / \mathrm{Py}^{10}$ $\mathrm{CH}_{2}=\mathrm{CHCH}_{2} \mathrm{Br} / \mathrm{CDI}$ (N,N'-carbonyldiimidazole), ${ }^{11} \mathrm{SOBr}_{2}{ }^{9}$ for bromination, MeI / $\mathrm{CDI}^{11}$ and $\mathrm{PI}_{3}{ }^{9}$ for iodination (Scheme 1). This methodology has been employed in the preparation of a variety of compounds, 
the range of yields is large (25-97\%) but the harsh reaction conditions, high temperatures and long reaction times preclude the presence of many functionalities.

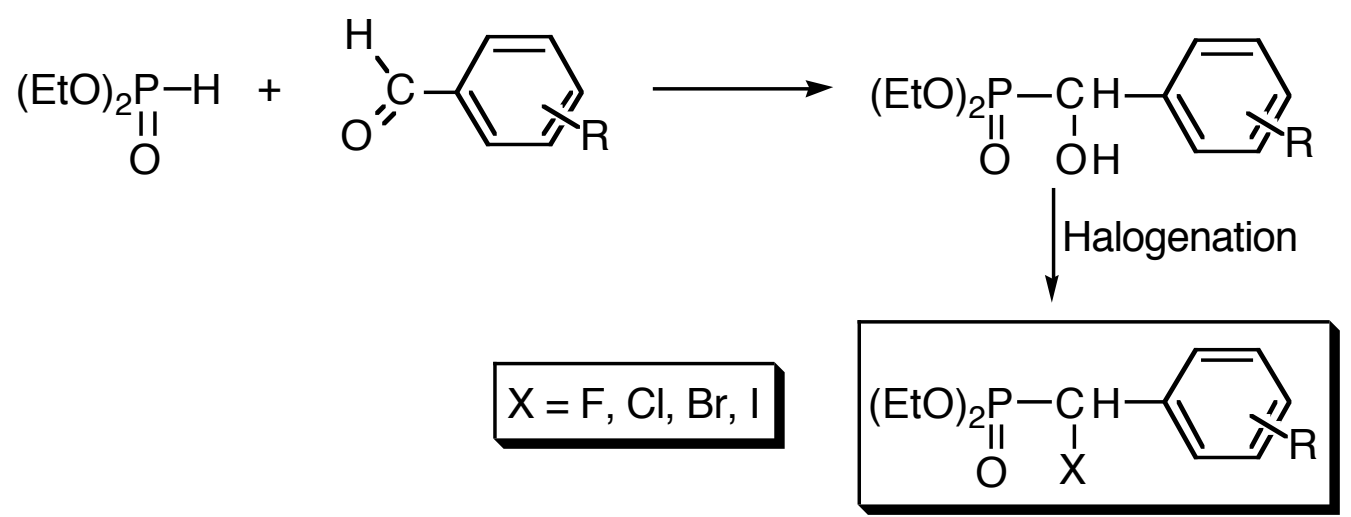

Scheme 1.

(b) Occasionally, the Michaelis-Arbuzov ${ }^{12}$ reaction may be used for the synthesis of $\alpha$ fluorobenzylphosphonate. This unusual approach proved to be problematic in the case of the secondary halides and the presence of an electron withdrawing group on the same carbon is needed to perform the reaction. ${ }^{13}$ Thus, bromofluorotoluene reacts with $(\mathrm{EtO})_{3} \mathrm{P}$ to afford diethyl $p$-carbethoxy- $\alpha$ fluorobenzylphosphonate in $68 \%$ yield as illustrated in Scheme 2. ${ }^{14}$

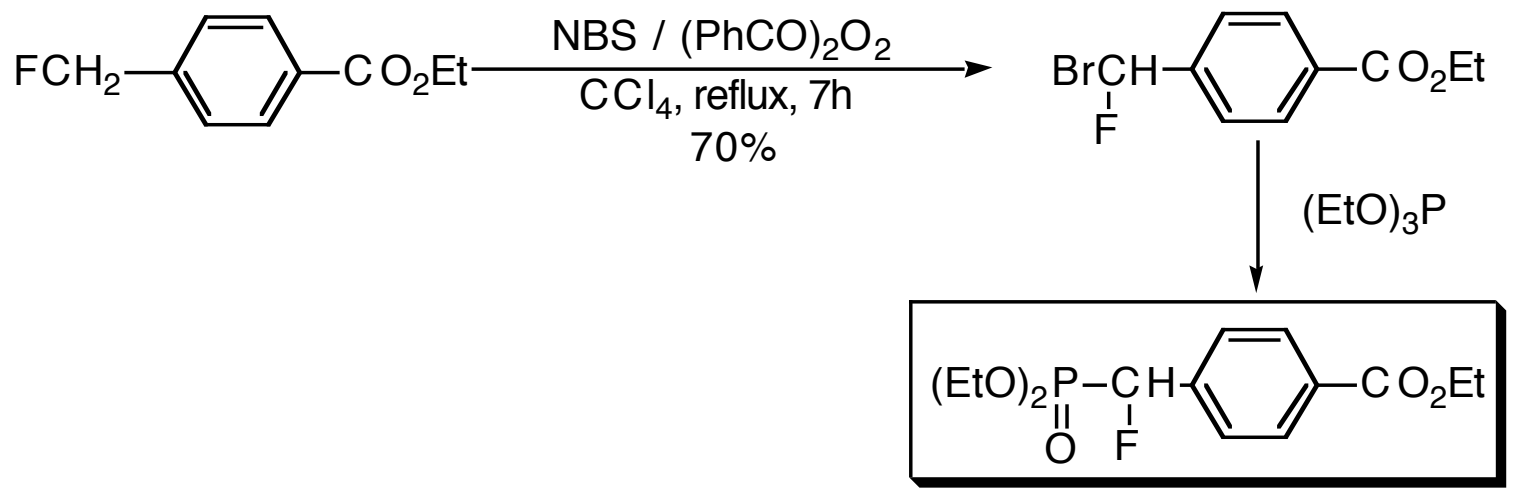

\section{Scheme 2.}

(c) Electrophilic chlorination of the diethyl $\alpha$-lithiobenzylphosphonate carbanion with $\mathrm{CCl}_{4}$ occured in moderate to good yields (82-90\%) (Scheme 3). According to the nature and position of the R group on the aromatic ring, the chlorination reaction was often sluggish. ${ }^{15}$
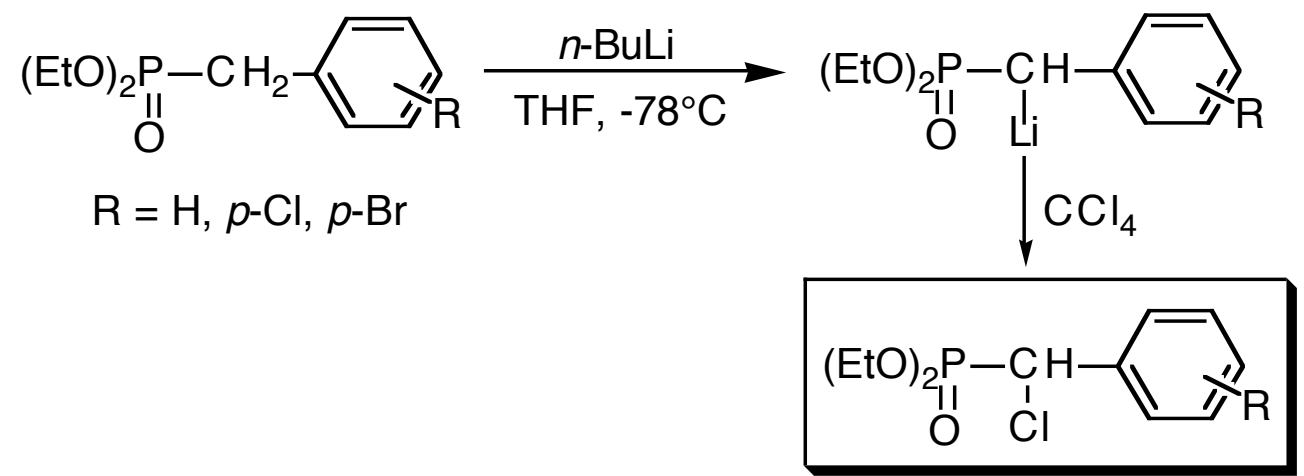

Scheme 3. 
(d) Only one example of preparation of diethyl $\alpha$-bromobenzylphosphonate by radical bromination using NBS in the presence of benzoyl peroxide was reported (Scheme 4). ${ }^{16}$ The reaction was very slow , requiring $\mathrm{CCl}_{4}$ at reflux and long reaction times (2-3 days), to give moderate yields (47-53\%).

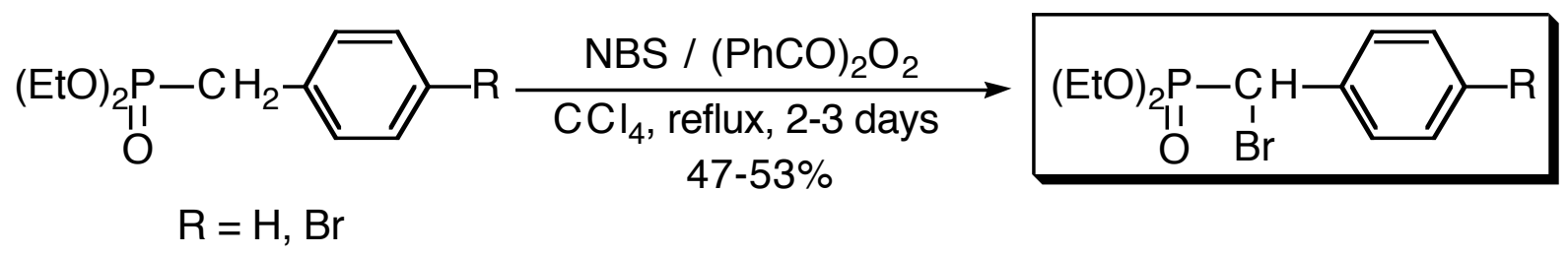

\section{Scheme 4.}

Despite recent advances in developing methodology and new halogenation reagents, these approaches are not highly selective and appear to have only limited synthetic potential. Consequently, mixtures of nonhalogenated and bis-halogenated benzylphosphonates could result, thereby canceling these reactions for synthetic purposes.

Despite a large number of investigations, the effective control of the electrophilic monohalogenation at the $\alpha-\mathrm{CH}_{2}$ position of phosphonates remains largely a delicate problem. The major disadvantage which attends the use of electrophilic approach is the formation of complex mixtures of monohalogeno-, dihalogeno- and nonhalogenobenzylphosphonates. Very recently, a wide variety of $\alpha, \alpha-$ difluorobenzylphosphonates have been synthesized from the corresponding phosphonates via electrophilic fluorination with NFBS. ${ }^{17}$ Owing to both biologic and synthetic potential of halogenobenzylphosphonates, the search of a reaction sequence that may be conveniently executed to produce a large variety of $\alpha$ monohalogenobenzylphosphonates in pure form is especially useful. The presence of protected carbanions is crucial to the success of the monohalogenation to exclude the formation of side reactions.

The purpose of this article is to report a new and highly selective access to $\alpha$-monohalogenobenzylphosphonates by controlled electrophilic halogenation of protected benzylphosphonate carbanions. Herein, the complete details of a general and attractive one-pot synthesis of diethyl $\alpha$-monofluoro-, chloro-, bromo-, and iodobenzylphosphonates are described. We performed our synthesis with a variety of diethyl benzylphosphonates which are commercially available or easily prepared on laboratory scale by heating $(\mathrm{EtO})_{3} \mathrm{P}$ with the corresponding benzyl halides according to the Michaelis-Arbuzov process. ${ }^{18-29}$

\section{RESULTS AND DISCUSSION}

The preparation of diethyl $\alpha$-monohalogenobenzylphosphonates $4(\mathrm{X}=\mathrm{F}), 5(\mathrm{X}=\mathrm{Cl}), \mathbf{6}(\mathrm{X}=\mathrm{Br})$ and 7 $(\mathrm{X}=\mathrm{I})$ from diethyl benzylphosphonates 1 is accomplished by a four steps, one-pot procedure via electrophilic halogenation of the 1-lithio-1-(trimethylsilyl)benzylphosphonates 2. Each step of this reaction sequence may be conveniently executed without isolation of the intermediates (Scheme 5). In the first step, extraction of a proton at the benzylic position from 1 occurs easily at low temperature with LiHMDS. This first deprotonation is followed at room temperature by simultaneous protection of the benzylic position with TMSCl and extraction of the second benzylic proton. The complete transformation of 1 into 2 needs 3 eq. of LiHMDS and the resulting silylated carbanions $\mathbf{2}$ are stable and easily identified by ${ }^{31} \mathrm{P}-\mathrm{NMR}$ analysis $\left(\delta^{31} \mathrm{P}(\mathrm{THF})=+47.3 \mathrm{ppm}\right)$. The use of a transient protection of the benzylic position generates a tertiary 
carbanion which reacts unambiguously with electrophile and thus avoids the problems associated with the formation of bis-halogenated derivatives.

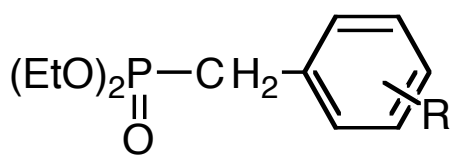

1
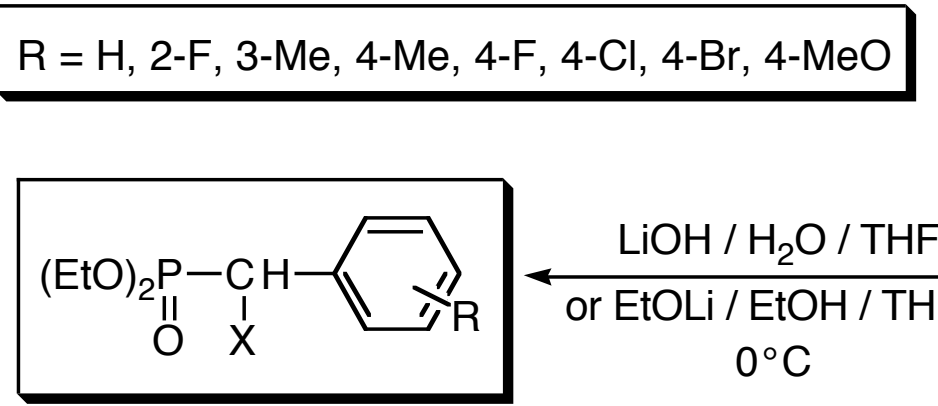

$4 a-h, X=F$

$5 a-h, X=C l$

6a-f, $X=B r$

$7 a-b, x=1$
1) 3 eq. LiHMDS / THF
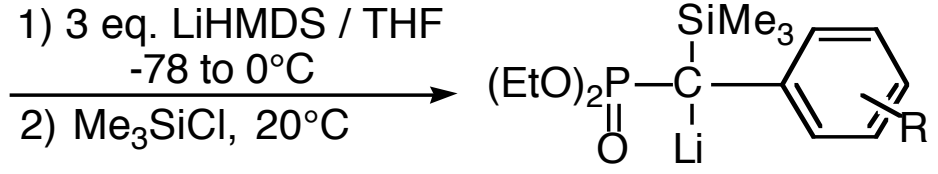

2

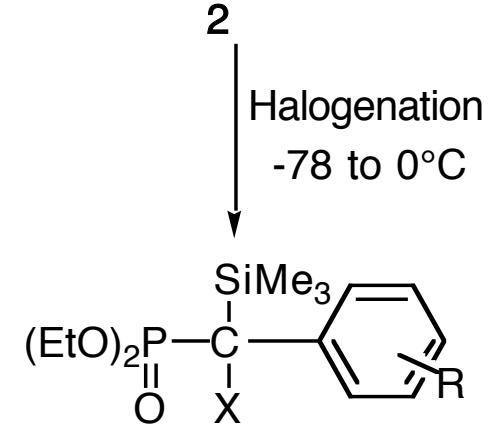

3

Scheme 5.

Halogenation of the tertiary carbanions $\mathbf{2}$ is effectively achieved using several electrophilic halogenating reagents, including $\mathrm{N}$-fluorobenzenesulfonimide (NFBS) for fluorination, hexachloroethane $\left(\mathrm{Cl}_{3} \mathrm{C}-\mathrm{CCl}_{3}\right)$ for chlorination, tetrachlorodibromoethane $\left(\mathrm{BrCl}_{2} \mathrm{C}-\mathrm{CCl}_{2} \mathrm{Br}\right)$ for bromination and iodine for iodination. All these reagents are crystalline, non-hygroscopic, easy to handle and commercially available. Furthermore, the chlorination and bromination reagents give reaction by-products which are volatile and easily eliminated. Chlorination, bromination and iodination, under various conditions of temperature, demonstrated identical reaction behaviour in the range $-90^{\circ} \mathrm{C}$ to $-20^{\circ} \mathrm{C}$. By contrast, fluorination has to be achieved exclusively at low temperature $\left(-90^{\circ} \mathrm{C}\right)$ to prevent aromatic ring fluorination, which is generally favoured by the presence of the electron-donating substituents. In these conditions the halogenation reactions are fast, clean and complete providing quantitative formation of $\mathbf{3}\left(\delta^{31} \mathrm{P}(\mathrm{THF})=+20.5 \mathrm{ppm}\right)$.

Direct elimination of the TMS group from 3 at $0^{\circ} \mathrm{C}$ is realized with unusual facility. When compounds 3 are merely treated in the reaction medium with either an aqueous solution of $\mathrm{LiOH}$ or an ethanolic solution of EtOLi, the diethyl $\alpha$-monohalogenobenzylphosphonates $4 \mathbf{a}-\mathbf{h}, \mathbf{5 a - h}, \mathbf{6 a - f}$ and 7a-b are obtained in a few minutes as the only products $\left(\delta^{31} \mathrm{P}(\mathrm{THF})=+16 \mathrm{ppm}\right)\left(\right.$ in ${ }^{31} \mathrm{P}-\mathrm{NMR}$ spectroscopy we observe a shift of about 4 ppm between the silylated and desilylated derivatives). The efficiency of the deprotection step is due to the three electron-withdrawing groups (phosphoryl, halogen and aromatic ring) which participate in the reaction by weakening the $\mathrm{C}$-Si bond and increasing the rate of desilylation. By contrast, deprotection of nonhalogenated diethyl trimethylsilylbenzylphosphonates is a slow reaction, requiring stirring overnight at room temperature or heating.

As is evident for a process involving several operations, experimental variables may affect the course of the reaction and we will discuss of the experimental parameters which have been recognized as important 
to succeed the reactions. These include: (a) the nature and quantity of the base; (b) the reaction temperature; (c) the stoichiometry of the reagents; (d) the removal conditions of the TMS group and (e) the influence of substituents on the aromatic ring.

(a) Three lithium amides, LiHMDS (lithium hexamethyldisilazide), LDA (lithium diisopropylamide) and LiTMP (lithium 2,2,6,6-tetramethylpiperidide) were tested as metallation agents. All three were basic enough to give the two successive deprotonations. However, we found that diisopropylamine and 2,2,6,6-tetramethylpiperidine, which are regenerated in the reaction mixture, are not inert towards TMSCl under our experimental conditions and react at room temperature with $\mathrm{TMSCl}$ forming $\mathrm{HCl}$ which partially protonates the carbanions 2 and comes back to the starting diethyl benzylphosphonates $\mathbf{1}$. By contrast hexamethyldisilazane is a more sterically hindered amine, less basic too, which is tolerated in the reaction of benzylic carbanions with TMSCl.

We proved that for a fast and complete obtaining of diethyl 1-lithio-1-(trimethylsilyl)benzylphosphonates 2 it is necessary to employ 3 eq. of base. Effectively, when a sample of diethyl 1(trimethylsilyl)benzylphosphonate is independently submitted to deprotonation with either LiHMDS or LDA at room temperature in THF, 2 eq. of base are required to provide full metallation.

(b) The reaction of $\mathrm{TMSCl}$ with the metallated derivative of $\mathbf{1}$ proceeds exclusively at room temperature. At low temperature the reaction is sluggish and never complete.

(c) TMSCl has to be introduced without excess to prevent side reactions with the free amine but above all because TMSCl undergoes an halogen exchange reaction with NFBS. In the presence of unreacted TMSCl, despite the use of an excess of NFBS, the fluorination reaction remains largely uncomplete.

(d) Two appropriate hydrolytic procedures may be employed for the cleavage of the C-Si bond. The method using $\mathrm{LiOH} / \mathrm{H}_{2} \mathrm{O}$ is preferred for fluorinated benzylphosphonates $3(\mathrm{X}=\mathrm{F})$ and the method using EtOLi / EtOH for chloro-, bromo- and iodobenzylphosphonates 3 (X=Cl, Br, I). In the case of $\mathbf{3}(\mathrm{X}=\mathrm{F})$, the cleavage of the $\mathrm{C}$-Si bond with ethanolic lithium ethoxide produces $\mathrm{N}$-ethylbenzenesulfonimide which remains in the organic phase and consequently is very difficult to separate from the $\alpha$ monofluorobenzylphosphonates 4a-f, whereas treatment of $\mathbf{3}(\mathrm{X}=\mathrm{F})$ with concentrated aqueous lithium hydroxyde affords lithium benzenesulfonimide which is retained in the aqueous phase. In addition, while no difficulties are encountered in the treatment of compounds 3 with lithium hydroxide, the use of sodium hydroxide is not appropriate for retaining benzesulfonimide in the aqueous phase.

(e) We also studied the influence of substituents $\mathrm{R}$ of the aromatic ring. The reaction is general for substituents such as $p$ - and $m$-methyl, $o$ - and $p$-fluoro, $p$-chloro, $p$-bromo and $p$-methoxy which do not interfere with the halogenation process. These substituents $\mathrm{R}$ have a significant influence when they are in the ortho-position. Under these conditions, the second deprotonation is slowed down because of a difficult approach of LiHMDS in a highly crowded position. Fluorine atom is the largest substituent allowed for successful deprotonation. This can be explained by the comparable size of fluorine and hydrogen atoms. The only cases where halogenation occurs are when the aromatic substituent $\mathrm{R}=2-\mathrm{F}$ (Scheme 5). All attempts to prepare $\alpha$-halogeno- $O$-chlorobenzylphosphonates in pure form failed.

All isolated compounds are stable at room temperature, except for the $\alpha$-iodobenzylphosphonates 7 which slowly decompose. 
The spectra of the $\alpha$-halogenobenzylphosphonates show a magnetic non-equivalence of the O,Odiethyl groups due to the presence of an asymmetric carbon in the $\alpha$-position inducing a diastereotopic effect at the phosphoryl group. This diastereotopic effect is not the consequence of a restricted rotation around the phosphorous-carbon bond. ${ }^{11}$ The main spectroscopic data are collected in the following Tables 1-4.

Table ${ }^{1} \cdot{ }^{31} \mathrm{P},{ }^{19} \mathrm{~F},{ }^{1} \mathrm{H},{ }^{13} \mathrm{C}$-NMR data of diethyl $\alpha$-fluorobenzylphosphonates 4

\begin{tabular}{|c|c|c|c|c|c|c|}
\hline 4 & $\mathrm{R}$ & $\begin{array}{c}\delta^{31} \mathrm{P}(\mathrm{ppm}) \\
\left(\mathrm{CDCl}_{3}\right)\end{array}$ & $\begin{array}{c}\delta^{19} \mathrm{~F}(\mathrm{ppm}) \\
\left(\mathrm{CDCl}_{3}\right)\end{array}$ & $\begin{array}{c}\delta{ }^{1} \mathrm{H} \text { P-CHF-Ar-R } \\
(\mathrm{ppm})\left(\mathrm{CDCl}_{3}\right)\end{array}$ & $\begin{array}{c}\delta{ }^{13} \mathrm{C} \text { P-CHF-Ar-R } \\
(\mathrm{ppm})\left(\mathrm{CDCl}_{3}\right)\end{array}$ & $\begin{array}{c}\text { Yields } \\
(\%)\end{array}$ \\
\hline $\mathbf{a}$ & $\mathrm{H}$ & $13.0\left(\mathrm{~d},{ }^{2} J_{\mathrm{PF}}=85.6\right)$ & $\left(\mathrm{d},{ }^{-200.6}{ }_{\mathrm{PF}}=85.6\right)$ & $\begin{array}{c}5.75\left(\mathrm{dd},{ }^{2} J_{\mathrm{PH}}=7.9\right. \\
\left.{ }^{2} J_{\mathrm{FH}}=44.6\right)\end{array}$ & $\begin{array}{c}89.9\left(\mathrm{dd},{ }^{1} J_{\mathrm{PC}}=170.5\right. \\
\left.{ }^{1} J_{\mathrm{FC}}=184.2\right)\end{array}$ & 97 \\
\hline b & $2-\mathrm{F}$ & $\begin{array}{c}12.6\left(\mathrm{dd},{ }^{2} J_{\mathrm{PF}}=88.6\right. \\
\left.{ }^{4} J_{\mathrm{PF}}=5.2\right)\end{array}$ & $\left(\mathrm{d},{ }^{-204.0}{ }_{\mathrm{PF}}=90.4\right)$ & $\begin{array}{c}6.10\left(\mathrm{dd},{ }^{2} J_{\mathrm{PH}}=8.0,\right. \\
\left.{ }^{2} J_{\mathrm{FH}}=44.2\right)\end{array}$ & $\begin{array}{l}83.5\left(\mathrm{ddd},{ }^{1} J_{\mathrm{PC}}=177.0\right. \\
\left.{ }^{1} J_{\mathrm{FC}}=180.1,{ }^{3} J_{\mathrm{FC}}=3.0\right)\end{array}$ & 68 \\
\hline c & 3-Me & $15.5\left(\mathrm{dd},{ }^{2} J_{\mathrm{PF}}=85.9\right)$ & $\left(\begin{array}{c}-198.4 \\
\left.{ }^{2} J_{\mathrm{PF}}=86.1\right)\end{array}\right.$ & $\begin{array}{c}5.75\left(\mathrm{dd},{ }^{2} J_{\mathrm{PH}}=7.5\right. \\
\left.{ }^{2} J_{\mathrm{FH}}=44.7\right)\end{array}$ & $\begin{array}{c}89.6\left(\mathrm{dd},{ }^{1} J_{\mathrm{PC}}=170.0\right. \\
\left.{ }^{1} J_{\mathrm{FC}}=183.7\right)\end{array}$ & 83 \\
\hline d & 4-Me & $13.5\left(\mathrm{~d},{ }^{2} J_{\mathrm{PF}}=87.7\right)$ & $\left(\begin{array}{c}-199.2 \\
\left({ }^{2} J_{\mathrm{PF}}=86.9\right)\end{array}\right.$ & $\begin{array}{c}5.70\left(\mathrm{dd},{ }^{2} J_{\mathrm{PH}}=7.5\right. \\
\left.{ }^{2} J_{\mathrm{FH}}=44.4\right)\end{array}$ & $\begin{array}{c}89.6\left(\mathrm{dd},{ }^{1} J_{\mathrm{PC}}=172.4\right. \\
\left.{ }^{1} J_{\mathrm{FC}}=183.2\right)\end{array}$ & 90 \\
\hline $\mathbf{e}$ & $4-\mathrm{F}$ & $12.9\left(\mathrm{~d},{ }^{2} J_{\mathrm{PF}}=85.6\right)$ & $\left(\begin{array}{c}-199.2 \\
\left({ }^{2} J_{\mathrm{PF}}=85.8\right)\end{array}\right.$ & $\begin{array}{c}5.62\left(\mathrm{dd},{ }^{2} J_{\mathrm{PH}}=7.5,\right. \\
\left.{ }^{2} J_{\mathrm{FH}}=44.5\right)\end{array}$ & $\begin{array}{c}88.7\left(\mathrm{dd},{ }^{1} J_{\mathrm{PC}}=171.5\right. \\
\left.{ }^{1} J_{\mathrm{FC}}=183.9\right)\end{array}$ & 68 \\
\hline $\mathbf{f}$ & $4-\mathrm{Cl}$ & $12.6\left(\mathrm{~d},{ }^{2} J_{\mathrm{PF}}=83.3\right)$ & $\left(\begin{array}{c}-201.4 \\
\left({ }^{2} J_{\mathrm{PF}}=83.6\right)\end{array}\right.$ & $\begin{array}{c}5.70\left(\mathrm{dd},{ }^{2} J_{\mathrm{PH}}=7.3\right. \\
\left.{ }^{2} J_{\mathrm{FH}}=44.5\right)\end{array}$ & $\begin{array}{c}88.9\left(\mathrm{dd},{ }^{1} J_{\mathrm{PC}}=172.9\right. \\
\left.{ }^{1} J_{\mathrm{FC}}=184.0\right)\end{array}$ & 74 \\
\hline g & $4-\mathrm{Br}$ & $14.4\left(\mathrm{~d},{ }^{2} J_{\mathrm{PF}}=85.1\right)$ & $\begin{array}{c}\left(\mathrm{d},{ }^{2}{ }_{\mathrm{PF}}=89.7\right. \\
=85.4)\end{array}$ & $\begin{array}{c}5.77\left(\mathrm{dd},{ }^{2} J_{\mathrm{PH}}=8.0,\right. \\
\left.{ }^{2} J_{\mathrm{FH}}=44.6\right)\end{array}$ & $\begin{array}{c}89.1\left(\mathrm{dd},{ }^{1} J_{\mathrm{PC}}=171.0,\right. \\
\left.{ }^{1} J_{\mathrm{FC}}=184.6\right)\end{array}$ & 76 \\
\hline $\mathbf{h}$ & 4-OMe & $15.8\left(\mathrm{dd},{ }^{2} J_{\mathrm{PF}}=91.3\right)$ & $\begin{array}{c}-192.5 \\
\left(\mathrm{~d},{ }^{2} J_{\mathrm{PF}}=90.3\right)\end{array}$ & $\begin{array}{c}5.66\left(\mathrm{dd},{ }^{2} J_{\mathrm{PH}}=7.0,\right. \\
\left.{ }^{2} J_{\mathrm{FH}}=44.4\right)\end{array}$ & $\begin{array}{c}89.5\left(\mathrm{ddd},{ }^{1} J_{\mathrm{PC}}=173.9,\right. \\
\left.{ }^{1} J_{\mathrm{FC}}=183.0\right)\end{array}$ & 79 \\
\hline
\end{tabular}

Table 2. ${ }^{31} \mathrm{P},{ }^{1} \mathrm{H},{ }^{13} \mathrm{C}$-NMR data of diethyl $\alpha$-chlorobenzylphosphonates 5

\begin{tabular}{|c|c|c|c|c|c|}
\hline 5 & $\mathrm{R}$ & $\begin{array}{c}\delta^{31} \mathrm{P}(\mathrm{ppm}) \\
\left(\mathrm{CDCl}_{3}\right) \\
\end{array}$ & $\begin{array}{c}\delta{ }^{1} \mathrm{H} \text { P-CHCl-Ar-R } \\
(\mathrm{ppm})\left(\mathrm{CDCl}_{3}\right)\end{array}$ & $\begin{array}{c}\delta{ }^{13} \mathrm{C} \mathrm{P}-\underline{\mathrm{CHCl}} \mathrm{H}-\mathrm{Ar}-\mathrm{R} \\
(\mathrm{ppm})\left(\mathrm{CDCl}_{3}\right)\end{array}$ & $\begin{array}{c}\text { Yields } \\
(\%)\end{array}$ \\
\hline $\mathbf{a}$ & $\mathrm{H}$ & $18.2(\mathrm{~s})$ & $4.87\left(\mathrm{~d},{ }^{2} J_{\mathrm{PH}}=14.1\right)$ & $53.2\left(\mathrm{~d},{ }^{1} J_{\mathrm{PC}}=159.6\right)$ & 93 \\
\hline b & $2-\mathrm{F}$ & $16.6(\mathrm{~s})$ & $5.20\left(\mathrm{~d},{ }^{2} J_{\mathrm{PH}}=14.3\right)$ & $45.0\left(\mathrm{~d},{ }^{1} J_{\mathrm{PC}}=163.3\right)$ & 92 \\
\hline c & 3-Me & $17.3(\mathrm{~s})$ & $4.93\left(\mathrm{~d},{ }^{2} J_{\mathrm{PH}}=14.0\right)$ & $53.9\left(\mathrm{~d},{ }^{1} J_{\mathrm{PC}}=160.0\right)$ & 95 \\
\hline d & 4-Me & $17.5(\mathrm{~s})$ & $4.90\left(\mathrm{~d},{ }^{2} J_{\mathrm{PH}}=13.9\right)$ & $53.3\left(\mathrm{~d},{ }^{1} J_{\mathrm{PC}}=161.2\right)$ & 90 \\
\hline $\mathbf{e}$ & $4-\mathrm{F}$ & $17.0(\mathrm{~s})$ & $4.75\left(\mathrm{~d},{ }^{2} J_{\mathrm{PH}}=14.1\right)$ & $53.1\left(\mathrm{~d},{ }^{1} J_{\mathrm{PC}}=160.6\right)$ & 94 \\
\hline $\mathbf{f}$ & $4-\mathrm{Cl}$ & $16.4(\mathrm{~s})$ & $4.79\left(\mathrm{~d},{ }^{2} J_{\mathrm{PH}}=14.3\right)$ & $53.3\left(\mathrm{~d},{ }^{1} J_{\mathrm{PC}}=160.2\right)$ & 93 \\
\hline $\mathbf{g}$ & $4-\mathrm{Br}$ & $16.4(\mathrm{~s})$ & $4.75\left(\mathrm{~d},{ }^{2} J_{\mathrm{PH}}=14.3\right)$ & $53.1\left(\mathrm{~d},{ }^{1} J_{\mathrm{PC}}=159.5\right)$ & 93 \\
\hline
\end{tabular}


h $\quad 4-\mathrm{OMe} \quad 17.6(\mathrm{~s})$

$4.93\left(\mathrm{~d},{ }^{2} J_{\mathrm{PH}}=13.7\right)$

$53.9\left(\mathrm{~d},{ }^{1} J_{\mathrm{PC}}=162.0\right)$

95

Table $3 \cdot{ }^{31} \mathrm{P},{ }^{1} \mathrm{H},{ }^{13} \mathrm{C}$-NMR data of diethyl $\alpha$-bromobenzylphosphonates 6

\begin{tabular}{cccccc}
\hline 6 & $\mathrm{R}$ & $\begin{array}{c}\delta{ }^{31} \mathrm{P}(\mathrm{ppm}) \\
\left(\mathrm{CDCl}_{3}\right)\end{array}$ & $\begin{array}{c}\delta{ }^{1} \mathrm{H} \mathrm{P}-\mathrm{C} \mathrm{HBr}-\mathrm{Ar}-\mathrm{R} \\
(\mathrm{ppm})\left(\mathrm{CDCl}_{3}\right)\end{array}$ & $\begin{array}{c}\delta{ }^{13} \mathrm{C} \mathrm{P}-\mathrm{CHBr}-\mathrm{Ar}-\mathrm{R} \\
(\mathrm{ppm})\left(\mathrm{CDCl}_{3}\right)\end{array}$ & $\begin{array}{c}\text { Yields } \\
(\%)\end{array}$ \\
\hline a & $\mathrm{H}$ & $17.3(\mathrm{~s})$ & $4.81\left(\mathrm{~d},{ }^{2} J_{\mathrm{PH}}=13.0\right)$ & $42.0\left(\mathrm{~d},{ }^{1}{ }_{\mathrm{PC}}=159.5\right)$ & 87 \\
b & $2-\mathrm{F}$ & $16.2(\mathrm{~s})$ & $5.20\left(\mathrm{~d},{ }^{2} J_{\mathrm{PH}}=13.7\right)$ & $33.9\left(\mathrm{~d},{ }^{1} J_{\mathrm{PC}}=164.5\right)$ & 88 \\
c & $4-\mathrm{Me}$ & $17.6(\mathrm{~s})$ & $4.87\left(\mathrm{~d},{ }^{2} J_{\mathrm{PH}}=12.8\right)$ & $41.9\left(\mathrm{~d},{ }^{1} J_{\mathrm{PC}}=161.2\right)$ & 85 \\
d & $4-\mathrm{F}$ & $17.4(\mathrm{~s})$ & $4.74\left(\mathrm{~d},{ }^{2} J_{\mathrm{PH}}=13.1\right)$ & $40.9\left(\mathrm{~d},{ }^{1} J_{\mathrm{PC}}=160.3\right)$ & 93 \\
e & $4-\mathrm{Cl}$ & $16.8(\mathrm{~s})$ & $4.76\left(\mathrm{~d},{ }^{2} J_{\mathrm{PH}}=13.2\right)$ & $40.9\left(\mathrm{~d},{ }^{1} J_{\mathrm{PC}}=160.0\right)$ & 95 \\
f & $4-\mathrm{Br}$ & $16.6(\mathrm{~s})$ & $4.70\left(\mathrm{~d},{ }^{2} J_{\mathrm{PH}}=13.2\right)$ & $41.0\left(\mathrm{~d},{ }^{1} J_{\mathrm{PC}}=159.1\right)$ & 92 \\
\hline
\end{tabular}

Table $4 .{ }^{31} \mathrm{P},{ }^{1} \mathrm{H},{ }^{13} \mathrm{C}$-NMR data of diethyl $\alpha$-iodobenzylphosphonates 7

\begin{tabular}{|c|c|c|c|c|c|}
\hline 7 & $\mathrm{R}$ & $\begin{array}{c}\delta^{31} \mathrm{P}(\mathrm{ppm}) \\
\left(\mathrm{CDCl}_{3}\right) \\
\end{array}$ & $\begin{array}{c}\delta^{1}{ }^{1} \mathrm{H} \text { P-C } \underline{\mathrm{HI}}-\mathrm{Ar}-\mathrm{R} \\
(\mathrm{ppm})\left(\mathrm{CDCl}_{3}\right)\end{array}$ & $\begin{array}{c}\delta^{\delta{ }^{13} \mathrm{C} \mathrm{P}-\underline{\mathrm{C}} \mathrm{HI}-\mathrm{Ar}-\mathrm{R}} \\
(\mathrm{ppm})\left(\mathrm{CDCl}_{3}\right)\end{array}$ & $\begin{array}{c}\text { Yields } \\
(\%)\end{array}$ \\
\hline $\mathbf{a}$ & $\mathrm{H}$ & $19.6(\mathrm{~s})$ & $4.96\left(\mathrm{~d},{ }^{2} \mathrm{~J}_{\mathrm{PH}}=13.5\right)$ & $15.4\left(\mathrm{~d},{ }^{1} \mathrm{~J}_{\mathrm{PC}}=155.6\right)$ & 98 \\
\hline b & 4-Me & $20.7(\mathrm{~s})$ & $5.09\left(\mathrm{~d},{ }^{2} \mathrm{~J}_{\mathrm{PH}}=13.5\right)$ & $16.2\left(\mathrm{~d},{ }^{1} \mathrm{~J}_{\mathrm{PC}}=160.2\right)$ & 97 \\
\hline
\end{tabular}

\section{CONCLUSION}

Consequently, we describe herein a general, versatile and unambiguous one-pot procedure for the synthesis of a wide variety of diethyl $\alpha$-monohalogenobenzylphosphonates in good yields via the electrophilic halogenation of the benzylphosphonate anions. The experimental conditions offer a shorter and more convenient preparation than those early reported, each step of this reaction sequence being executed in quantitative yields without the isolation of intermediates. Extension of this methodology, using easily available electrophilic halogenation reagents, to other functional phosphonates is in progress.

\section{EXPERIMENTAL SECTION}

NMR spectra were recorded on a Bruker AC 200 spectrometer operating at $200 \mathrm{MHz}$ for proton, 50.3 $\mathrm{MHz}$ for carbon and $81.01 \mathrm{MHz}$ for phosphorus. ${ }^{31} \mathrm{P}$ downfield shifts $(\delta)$ are expressed with a positive sign, in ppm, relative to external $85 \% \mathrm{H}_{3} \mathrm{PO}_{4}$ in $\mathrm{H}_{2} \mathrm{O} .{ }^{1} \mathrm{H}$ and ${ }^{13} \mathrm{C}$ chemical shifts $(\delta)$ are reported in ppm relative to $\mathrm{CDCl}_{3}$ as internal standard. ${ }^{19} \mathrm{~F}$ chemical shifts $(\delta)$ are reported in ppm relative to $\mathrm{CFCl}_{3}$ as external standard. Coupling constants $(J)$ are given in Hz. Gas chromatography was performed on a Hewlett Packard 5890 gas spectrometer with an SGE BPX $(25 \mathrm{M} \times 0.22 \mathrm{~mm})$ column using splitless injection and a helium gas vector at $1 \mathrm{ml} / \mathrm{min}$. Low resolution mass spectra were recorded on a Hewlet Packard 5989 B mass spectrometer. 
Organic solvents were purified by standard procedures. THF was distilled under an inert atmosphere from purple solutions of sodium benzophenone ketyl. The synthesis of all compounds were carried out under dry nitrogen.

General procedure for the synthesis of diethyl benzylphosphonates

An oven-dried, $500 \mathrm{ml}$, four necked, round-bottomed flask was fitted with an efficient mechanical stirrer, a thermometer, a fractionating Vigreux column equipped with a condenser, and a pressure equalizing funnel with a nitrogen inlet. Under a gentle flow of nitrogen the flask was charged with 0.4 mol of a substituted benzyl chloride or bromide. The flask was heated at $150^{\circ} \mathrm{C}$ (for benzyl chloride) or at $100^{\circ} \mathrm{C}$ (for benzyl bromide). At this temperature $66.4 \mathrm{~g}(0.4 \mathrm{~mol})$ of $(\mathrm{EtO})_{3} \mathrm{P}$ was slowly added. After the addition was completed, the mixture was heated for $5 \mathrm{~h}$ at $150^{\circ} \mathrm{C}$ for benzyl chloride and for $2 \mathrm{~h}$ at $100^{\circ} \mathrm{C}$ for benzyl bromide. The reaction is easily monitored by ${ }^{31} \mathrm{P}-\mathrm{NMR}$ spectroscopy. When all the ethyl bromide was distilled, in the case of benzyl bromide, the residue was transferred to a pear-shaped flask for distillation through a Vigreux column and fractionated at vacuum-pump pressure.

Diethyl benzylphosphonate $\quad(\mathbf{1 a})^{18-23} \mathrm{C}_{11} \mathrm{H}_{17} \mathrm{O}_{3} \mathrm{P}\left(\mathrm{M=228)}:{ }^{31} \mathbf{P}-\mathbf{N M R}+27.4\right.$ (s). / 1 H-NMR 1.17 (t, $\left.{ }^{3} J_{\mathrm{HH}}=7.1,6 \mathrm{H}, \mathrm{CH}_{3} \mathrm{CH}_{2} \mathrm{O}\right), 3.09\left(\mathrm{~d},{ }^{2} J_{\mathrm{PH}}=21.6,2 \mathrm{H}, \mathrm{PCH}_{2}\right), 3.94\left(\mathrm{p},{ }^{3} J_{\mathrm{HH}}={ }^{3} J_{\mathrm{PH}}=7.1,4 \mathrm{H}, \mathrm{CH}_{3} \mathrm{CH}_{2} \mathrm{O}\right), 7.16-$ $7.24\left(\mathrm{~m}, 5 \mathrm{H}, \underline{\mathrm{H}}_{\mathrm{arom}}\right.$ of $\left.\mathrm{C}_{6} \mathrm{H}_{5}\right) . /{ }^{13} \mathrm{C}-\mathrm{NMR} 16.8\left(\mathrm{~d},{ }^{3} \mathrm{~J}_{\mathrm{PC}}=5.8, \underline{\mathrm{CH}}_{3} \mathrm{CH}_{2} \mathrm{O}\right), 34.3\left(\mathrm{~d},{ }^{1} J_{\mathrm{PC}}=138.5, \underline{\mathrm{CH}}_{2}\right), 62.6$ $\left(\mathrm{d},{ }^{2} J_{\mathrm{PC}}=7.0, \mathrm{CH}_{3} \mathrm{CH}_{2} \mathrm{O}\right), 127.3\left(\mathrm{~d},{ }^{5} \mathrm{~J}_{\mathrm{PC}}=3.0, \underline{\mathrm{C}}_{\text {para }}\right.$ of $\left.\mathrm{C}_{6} \mathrm{H}_{5}\right), 129.0\left(\mathrm{~d},{ }^{4} J_{\mathrm{PC}}=2.7, \underline{\mathrm{C}}_{\text {meta }}\right.$ of $\left.\mathrm{C}_{6} \mathrm{H}_{5}\right), 130.3(\mathrm{~d}$, ${ }^{3} J_{\mathrm{PC}}=7.1, \underline{\mathrm{C}}_{\text {ortho }}$ of $\left.\mathrm{C}_{6} \mathrm{H}_{5}\right), 132.1\left(\mathrm{~d},{ }^{2} J_{\mathrm{PC}}=9.3, \underline{\mathrm{C}}_{\mathrm{ipso}}\right.$ of $\left.\mathrm{C}_{6} \mathrm{H}_{5}\right)$. / m/z $(\mathrm{CI}): 229(\mathrm{M}+1,100), 246(\mathrm{M}+18,11)$.

Diethyl 2-fluorobenzylphosphonate $(\mathbf{1 b})^{18,24,25} \mathrm{C}_{11} \mathrm{H}_{16} \mathrm{O}_{3} \mathrm{FP}(\mathrm{M}=246)$ : 31P-NMR +24.8 (s). / 19F-NMR 90.7 (s). / 1 $\mathbf{H}-\mathbf{N M R} 1.09\left(\mathrm{t},{ }^{3} J_{\mathrm{HH}}=7.0,6 \mathrm{H}, \mathrm{CH}_{3} \mathrm{CH}_{2} \mathrm{O}\right), 3.04$ (d, $\left.{ }^{2} J_{\mathrm{PH}}=21.6,2 \mathrm{H}, \mathrm{PC}_{2}\right), 3.89$ (p, $\left.{ }^{3} J_{\mathrm{HH}}={ }^{3} J_{\mathrm{PH}}=7.0,4 \mathrm{H}, \mathrm{CH}_{3} \underline{\mathrm{CH}}_{2} \mathrm{O}\right), 6.83-7.27\left(\mathrm{~m}, 4 \mathrm{H}, \underline{\mathrm{H}}_{\text {arom }}\right.$ of $\left.\mathrm{C}_{6} \mathrm{H}_{4}\right)$. / 13C-NMR $16.6\left(\mathrm{~d},{ }^{3} J_{\mathrm{PC}}=6.1\right.$, $\left.\mathrm{CH}_{3} \mathrm{CH}_{2} \mathrm{O}\right), 26.6\left(\mathrm{dd},{ }^{1} J_{\mathrm{PC}}=140.4,{ }^{3} J_{\mathrm{FC}}=2.7, \mathrm{PCH}_{2}\right), 62.5\left(\mathrm{~d},{ }^{2} J_{\mathrm{PC}}=7.1, \mathrm{CH}_{3} \underline{C H}_{2} \mathrm{O}\right), 115.6\left(\mathrm{dd},{ }^{4} J_{\mathrm{PC}}=2.8\right.$, ${ }^{2} J_{\mathrm{FC}}=22.8, \underline{\mathrm{C}}_{\text {meta }}$ of $\left.\mathrm{C}_{6} \mathrm{H}_{4}\right), 119.4\left(\mathrm{dd},{ }^{2} J_{\mathrm{PC}}=16.0,{ }^{2} J_{\mathrm{FC}}=9.7, \underline{\mathrm{C}}_{\mathrm{ipso}}\right.$ of $\left.\mathrm{C}_{6} \mathrm{H}_{4}\right), 124.4\left(\mathrm{t},{ }^{4} J_{\mathrm{PC}}={ }^{4} J_{\mathrm{FC}}=3.6, \underline{\mathrm{C}}_{\text {meta' }}\right.$ of $\left.\mathrm{C}_{6} \mathrm{H}_{4}\right), 129.0\left(\mathrm{dd},{ }^{5} J_{\mathrm{PC}}=3.7,{ }^{3} J_{\mathrm{FC}}=7.7, \underline{\mathrm{C}}_{\mathrm{para}}\right.$ of $\left.\mathrm{C}_{6} \mathrm{H}_{4}\right), 132.1\left(\mathrm{t},{ }^{3} J_{\mathrm{PC}}={ }^{3} J_{\mathrm{FC}}=4.4, \underline{\mathrm{C}}_{\text {ortho' }}\right.$ of $\left.\mathrm{C}_{6} \mathrm{H}_{4}\right), 161.1$ $\left(\mathrm{dd},{ }^{3} J_{\mathrm{PC}}=7.6,{ }^{1} J_{\mathrm{FC}}=246.9, \underline{\mathrm{C}}_{\text {ortho }}\right.$ of $\left.\mathrm{C}_{6} \mathrm{H}_{4}\right) . / \mathbf{m} / \mathbf{z}(\mathrm{CI}): 247(\mathrm{M}+1,100), 264(\mathrm{M}+18,63)$.

Diethyl 2-chlorobenzylphosphonate (1c) ${ }^{26,29} \mathrm{C}_{11} \mathrm{H}_{16} \mathrm{O}_{3} \mathrm{ClP}(\mathrm{M}=262.5)$ : 31P-NMR +26.1 (s). / 1H-NMR $1.14\left(\mathrm{t},{ }^{3} J_{\mathrm{HH}}=7.0,6 \mathrm{H}, \mathrm{CH}_{3} \mathrm{CH}_{2} \mathrm{O}\right), 3.26\left(\mathrm{~d},{ }^{2} J_{\mathrm{PH}}=22.0,2 \mathrm{H}, \mathrm{PC}_{2}\right), 3.90\left(\mathrm{p},{ }^{3} J_{\mathrm{HH}}={ }^{3} J_{\mathrm{PH}}=7.0,4 \mathrm{H}\right.$, $\left.\mathrm{CH}_{3} \underline{\mathrm{C}}_{2} \mathrm{O}\right)$, 7.04-7.36 (m, 4H, $\underline{\mathrm{H}}_{\mathrm{arom}}$ of $\left.\mathrm{C}_{6} \mathrm{H}_{4}\right)$. / ${ }^{13} \mathbf{C}-\mathrm{NMR}\left(\mathrm{CDCl}_{3}\right), \delta(\mathrm{ppm}): 16.5\left(\mathrm{~d},{ }^{3} J_{\mathrm{PC}}=6.1\right.$, $\left.\underline{\mathrm{CH}}_{3} \mathrm{CH}_{2} \mathrm{O}\right), 30.9\left(\mathrm{~d},{ }^{1} J_{\mathrm{PC}}=139.2, \mathrm{PCH}_{2}\right), 62.4\left(\mathrm{~d},{ }^{2} J_{\mathrm{PC}}=6.3, \mathrm{CH}_{3} \underline{\mathrm{CH}_{2} \mathrm{O}}\right), 127.0\left(\mathrm{~d},{ }^{5} J_{\mathrm{PC}}=4.0, \underline{\mathrm{C}}_{\mathrm{para}}\right.$ of $\left.\mathrm{C}_{6} \mathrm{H}_{4}\right)$, $128.5\left(\mathrm{~d}^{4} J_{\mathrm{PC}}=3.2, \underline{\mathrm{C}}_{\text {meta' }}\right.$ of $\left.\mathrm{C}_{6} \mathrm{H}_{4}\right), 129.8\left(\mathrm{~d},{ }^{4} J_{\mathrm{PC}}=2.9, \underline{\mathrm{C}}_{\text {meta }}\right.$ of $\left.\mathrm{C}_{6} \mathrm{H}_{4}\right), 130.2\left(\mathrm{~d},{ }^{2} J_{\mathrm{PC}}=9.2, \underline{\mathrm{C}}_{\text {ipso }}\right.$ of $\left.\mathrm{C}_{6} \mathrm{H}_{4}\right)$, $131.9\left(\mathrm{~d},{ }^{3} J_{\mathrm{PC}}=5.8, \underline{\mathrm{C}}_{\text {ortho' }}\right.$ of $\left.\mathrm{C}_{6} \mathrm{H}_{4}\right), 134.4\left(\mathrm{~d},{ }^{3} J_{\mathrm{PC}}=7.8, \underline{\mathrm{C}}_{\text {ortho }}\right.$ of $\left.\mathrm{C}_{6} \mathrm{H}_{4}\right) . / \mathbf{m} / \mathbf{z}(\mathrm{CI}): 263\left(\mathrm{M}+1{ }^{35} \mathrm{Cl}, 100\right)$, $265\left(\mathrm{M}+1{ }^{37} \mathrm{Cl}, 33\right), 280\left(\mathrm{M}+18{ }^{35} \mathrm{Cl}, 54\right), 282\left(\mathrm{M}+18{ }^{37} \mathrm{Cl}, 19\right)$.

Diethyl 3-methylbenzylphosphonate (1d) ${ }^{18,29} \mathrm{C}_{12} \mathrm{H}_{19} \mathrm{O}_{3} \mathrm{P}(\mathrm{M=}=242)$ : 31P-NMR +26.7 (s). / 1 H-NMR $1.12(\mathrm{t}$, $\left.{ }^{3} J_{\mathrm{HH}}=7.0,6 \mathrm{H}, \mathrm{C}_{3} \mathrm{CH}_{2} \mathrm{O}\right), 2.21\left(\mathrm{~d},{ }^{4} J_{\mathrm{HH}}=2.2,3 \mathrm{H}, \mathrm{C}_{6} \mathrm{H}_{4} \mathrm{C}_{3}\right), 2.98\left(\mathrm{~d},{ }^{2} J_{\mathrm{PH}}=21.6,2 \mathrm{H}, \mathrm{PC}_{2}\right), 3.89(\mathrm{p}$, $\left.{ }^{3} J_{\mathrm{HH}}={ }^{3} J_{\mathrm{PH}}=7.1,4 \mathrm{H}, \mathrm{CH}_{3} \underline{\mathrm{H}}_{2} \mathrm{O}\right), 6.90-7.11\left(\mathrm{~m}, 4 \mathrm{H}, \underline{\mathrm{H}}\right.$ of $\left.\mathrm{C}_{6} \mathrm{H}_{4}\right) . /{ }^{13} \mathrm{C}-\mathrm{NMR} 16.7\left(\mathrm{~d},{ }^{3} J_{\mathrm{PC}}=6.2, \underline{\mathrm{CH}}_{3} \mathrm{CH}_{2} \mathrm{O}\right)$, $21.6\left(\mathrm{~s}, \mathrm{C}_{6} \mathrm{H}_{4} \underline{\mathrm{CH}}_{3}\right), 33.9\left(\mathrm{~d},{ }^{1} J_{\mathrm{PC}}=137.5, \mathrm{PCH}_{2}\right), 62.3\left(\mathrm{~d},{ }^{2} J_{\mathrm{PC}}=6.9, \mathrm{CH}_{3} \underline{\mathrm{CH}}_{2} \mathrm{O}\right), 127.1\left(\mathrm{~d},{ }^{3} J_{\mathrm{PC}}=6.4, \underline{\mathrm{C}}_{\text {ortho }}\right.$ of $\left.\mathrm{C}_{6} \mathrm{H}_{4}\right), 127.9\left(\mathrm{~d},{ }^{5} J_{\mathrm{PC}}=4.4, \underline{\mathrm{C}}_{\text {para }}\right.$ of $\left.\mathrm{C}_{6} \mathrm{H}_{4}\right), 128.6\left(\mathrm{~d},{ }^{4} J_{\mathrm{PC}}=3.3, \underline{\mathrm{C}}_{\text {meta' }}\right.$ of $\left.\mathrm{C}_{6} \mathrm{H}_{4}\right), 130.8\left(\mathrm{~d},{ }^{3} J_{\mathrm{PC}}=7.3, \underline{\mathrm{C}}_{\text {ortho }}\right.$ of 
$\left.\mathrm{C}_{6} \mathrm{H}_{4}\right), 131.7\left(\mathrm{~d},{ }^{2} J_{\mathrm{PC}}=9.3, \underline{\mathrm{C}}_{\mathrm{ipso}}\right.$ of $\left.\mathrm{C}_{6} \mathrm{H}_{4}\right), 138.3\left(\mathrm{~d},{ }^{4} J_{\mathrm{PC}}=4.4, \underline{\mathrm{C}}_{\text {meta }}\right.$ of $\left.\mathrm{C}_{6} \mathrm{H}_{4}\right) . / \mathbf{m} / \mathbf{z}(\mathrm{CI}): 243(\mathrm{M}+1,100)$, $260(\mathrm{M}+18,7)$.

Diethyl 4-methylbenzylphosphonate (1e) $\mathrm{C}_{12} \mathrm{H}_{19} \mathrm{O}_{3} \mathrm{P}(\mathrm{M}=242)$ : 31P-NMR +26.3 (s). / 1 H-NMR 1.16 (t, $\left.{ }^{3} J_{\mathrm{HH}}=7.1,6 \mathrm{H}, \mathrm{CH}_{3} \mathrm{CH}_{2} \mathrm{O}\right), 2.24\left(\mathrm{~d},{ }^{4} J_{\mathrm{HH}}=2.2,3 \mathrm{H}, \mathrm{C}_{6} \mathrm{H}_{4} \mathrm{CH}_{3}\right), 3.03\left(\mathrm{~d},{ }^{2} J_{\mathrm{PH}}=21.4,2 \mathrm{H}, \mathrm{PC}_{2}\right), 3.93(\mathrm{p}$, $\left.{ }^{3} J_{\mathrm{HH}}={ }^{3} J_{\mathrm{PH}}=7.1,4 \mathrm{H}, \mathrm{CH}_{3} \underline{\mathrm{H}}_{2} \mathrm{O}\right), 7.03\left(\mathrm{~d},{ }^{3} J_{\mathrm{HH}}=8.2,2 \mathrm{H}, \underline{\mathrm{H}}_{\text {meta }}\right.$ of $\left.\mathrm{C}_{6} \mathrm{H}_{4}\right), 7.11\left(\mathrm{dd},{ }^{3} J_{\mathrm{HH}}=8.2,{ }^{4} J_{\mathrm{PH}}=2.2,2 \mathrm{H}\right.$, $\underline{\mathrm{H}}_{\text {para }}$ of $\left.\mathrm{C}_{6} \mathrm{H}_{4}\right) . /{ }^{13} \mathrm{C}-\mathrm{NMR} 16.8\left(\mathrm{~d},{ }^{3} \mathrm{~J}_{\mathrm{PC}}=5.6, \underline{\mathrm{CH}}_{3} \mathrm{CH}_{2} \mathrm{O}\right), 21.5\left(\mathrm{~s}, \mathrm{C}_{6} \mathrm{H}_{4} \underline{\mathrm{CH}}_{3}\right), 33.7\left(\mathrm{~d},{ }^{1} J_{\mathrm{PC}}=138.5, \mathrm{PCH}_{2}\right)$, $62.5\left(\mathrm{~d},{ }^{2} J_{\mathrm{PC}}=6.4, \mathrm{CH}_{3} \underline{\mathrm{CH}}_{2} \mathrm{O}\right), 128.8\left(\mathrm{~d},{ }^{2} J_{\mathrm{PC}}=9.2, \underline{\mathrm{C}}_{\mathrm{ipso}}\right.$ of $\left.\mathrm{C}_{6} \mathrm{H}_{4}\right), 129.7\left(\mathrm{~d},{ }^{4} J_{\mathrm{PC}}=2.8, \underline{\mathrm{C}}_{\mathrm{meta}}\right.$ of $\left.\mathrm{C}_{6} \mathrm{H}_{4}\right), 130.1$ $\left(\mathrm{d},{ }^{3} J_{\mathrm{PC}}=7.0, \underline{\mathrm{C}}_{\text {ortho }}\right.$ of $\left.\mathrm{C}_{6} \mathrm{H}_{4}\right), 136.8\left(\mathrm{~d},{ }^{5} \mathrm{~J}_{\mathrm{PC}}=5.4, \underline{\mathrm{C}}_{\text {para }}\right.$ of $\left.\mathrm{C}_{6} \mathrm{H}_{4}\right) . / \mathbf{m} / \mathbf{z}(\mathrm{CI}): 243(\mathrm{M}+1,100), 260(\mathrm{M}+18$, 13).

Diethyl 4-fluorobenzylphosphonate (1f) $\mathrm{C}_{11} \mathrm{H}_{16} \mathrm{O}_{3} \mathrm{FP}(\mathrm{M}=246)$ : 31P-NMR +26.0 (d, $\left.{ }^{6} J_{\mathrm{PF}}=5.4\right)$. / 19F-NMR $90.8\left(\mathrm{~d},{ }^{6} J_{\mathrm{PF}}=5.5\right) . /{ }^{\mathbf{1}} \mathbf{H}-\mathrm{NMR} 1.17\left(\mathrm{t},{ }^{3} \mathrm{~J}_{\mathrm{HH}}=7.0,6 \mathrm{H}, \mathrm{CH}_{3} \mathrm{CH}_{2} \mathrm{O}\right), 3.04\left(\mathrm{~d},{ }^{2} J_{\mathrm{PH}}=21.4,2 \mathrm{H}, \mathrm{PCH}_{2}\right), 3.94(\mathrm{p}$, $\left.{ }^{3} J_{\mathrm{HH}}={ }^{3} J_{\mathrm{PH}}=7.0,4 \mathrm{H}, \mathrm{CH}_{3} \underline{\mathrm{C}}_{2} \mathrm{O}\right), 6.92\left(\mathrm{t},{ }^{3} J_{\mathrm{HH}}={ }^{3} J_{\mathrm{FH}}=8.7,2 \mathrm{H}, \underline{\mathrm{H}}_{\text {meta }}\right.$ of $\left.\mathrm{C}_{6} \mathrm{H}_{4}\right), 7.14-7.23\left(\mathrm{~m}, 2 \mathrm{H}, \underline{\mathrm{H}}_{\mathrm{ortho}}\right.$ of $\left.\mathrm{C}_{6} \mathrm{H}_{4}\right)$. / 13C-NMR $16.9\left(\mathrm{~d},{ }^{3} J_{\mathrm{PC}}=6.0, \underline{\mathrm{CH}}_{3} \mathrm{CH}_{2} \mathrm{O}\right), 33.4\left(\mathrm{~d},{ }^{1} J_{\mathrm{PC}}=139.1, \mathrm{PCH}_{2}\right), 62.7\left(\mathrm{~d},{ }^{2} J_{\mathrm{PC}}=6.9\right.$, $\left.\mathrm{CH}_{3} \underline{\mathrm{CH}}_{2} \mathrm{O}\right), 116.0\left(\mathrm{dd},{ }^{4} J_{\mathrm{PC}}=2.7,{ }^{2} J_{\mathrm{FC}}=21.4, \underline{\mathrm{C}}_{\text {meta }}\right.$ of $\left.\mathrm{C}_{6} \mathrm{H}_{4}\right), 128.0\left(\mathrm{dd},{ }^{2} J_{\mathrm{PC}}=9.2,{ }^{4} J_{\mathrm{FC}}=3.0, \underline{\mathrm{C}}_{\mathrm{ipso}}\right.$ of $\left.\mathrm{C}_{6} \mathrm{H}_{4}\right)$, $131.8\left(\mathrm{t},{ }^{3} J_{\mathrm{PC}}={ }^{3} J_{\mathrm{FC}}=7.6, \underline{\mathrm{C}}_{\text {ortho }}\right.$ of $\left.\mathrm{C}_{6} \mathrm{H}_{4}\right), 162.5\left(\mathrm{dd},{ }^{5} J_{\mathrm{PC}}=4.6,{ }^{1} J_{\mathrm{FC}}=245.7, \underline{\mathrm{C}}_{\text {para }}\right.$ of $\left.\mathrm{C}_{6} \mathrm{H}_{4}\right) . / \mathbf{m} / \mathbf{z}(\mathrm{CI}): 247$ $(\mathrm{M}+1,100), 264(\mathrm{M}+18,22)$.

Diethyl 4-chlorobenzylphosphonate (1g) ${ }^{18,26} \mathrm{C}_{11} \mathrm{H}_{16} \mathrm{O}_{3} \mathrm{ClP}(\mathrm{M}=262.5)$ : 31P-NMR +25.3 (s). / 1H-NMR $1.24\left(\mathrm{t},{ }^{3} J_{\mathrm{HH}}=7.0,6 \mathrm{H}, \mathrm{C}_{3} \mathrm{CH}_{2} \mathrm{O}\right), 3.11\left(\mathrm{~d},{ }^{2} J_{\mathrm{PH}}=21.6,2 \mathrm{H}, \mathrm{PC}_{2}\right), 4.03\left(\mathrm{p},{ }^{3} J_{\mathrm{HH}}={ }^{3} J_{\mathrm{PH}}=7.0,4 \mathrm{H}\right.$, $\left.\mathrm{CH}_{3} \underline{\mathrm{C}}_{2} \mathrm{O}\right), 7.23-7.35\left(\mathrm{~m}, 4 \mathrm{H}, \underline{\mathrm{H}}_{\mathrm{arom}}\right.$ of $\left.\mathrm{C}_{6} \mathrm{H}_{4}\right)$. / 13 C-NMR $16.4\left(\mathrm{~d},{ }^{3} \mathrm{~J}_{\mathrm{PC}}=6.0, \underline{\mathrm{CH}}_{3} \mathrm{CH}_{2} \mathrm{O}\right), 33.1(\mathrm{~d}$, $\left.{ }^{1} J_{\mathrm{PC}}=138.0, \underline{\mathrm{PCH}}_{2}\right), 62.1\left(\mathrm{~d},{ }^{2} J_{\mathrm{PC}}=7.2, \mathrm{CH}_{3} \underline{\mathrm{CH}}_{2} \mathrm{O}\right), 128.6\left(\mathrm{~d},{ }^{4} J_{\mathrm{PC}}=2.8, \underline{\mathrm{C}}_{\text {meta }}\right.$ of $\left.\mathrm{C}_{6} \mathrm{H}_{4}\right), 130.4\left(\mathrm{~d},{ }^{2} J_{\mathrm{PC}}=9.2\right.$, $\underline{\mathrm{C}}_{\mathrm{ipso}}$ of $\left.\mathrm{C}_{6} \mathrm{H}_{4}\right), 131.1\left(\mathrm{~d},{ }^{3} J_{\mathrm{PC}}=6.2, \underline{\mathrm{C}}_{\text {ortho }}\right.$ of $\left.\mathrm{C}_{6} \mathrm{H}_{4}\right), 132.7\left(\mathrm{~d},{ }^{5} J_{\mathrm{PC}}=4.5, \underline{\mathrm{C}}_{\text {para }}\right.$ of $\left.\mathrm{C}_{6} \mathrm{H}_{4}\right) . / \mathbf{m} / \mathbf{z}(\mathrm{CI}): 263$ $\left(\mathrm{M}+1{ }^{35} \mathrm{Cl}, 100\right), 265\left(\mathrm{M}+1{ }^{37} \mathrm{Cl}, 38\right), 280\left(\mathrm{M}+18{ }^{35} \mathrm{Cl}, 27\right), 282\left(\mathrm{M}+18{ }^{37} \mathrm{Cl}, 9\right)$.

Diethyl 4-bromobenzylphosphonate $(\mathbf{1 h})^{27,28} \mathrm{C}_{11} \mathrm{H}_{16} \mathrm{O}_{3} \mathrm{BrP}(\mathrm{M}=307)$ : 31P-NMR + 20.1 (s). / 1 H-NMR 1.07 $\left(\mathrm{t},{ }^{3} J_{\mathrm{HH}}=7.0,6 \mathrm{H}, \mathrm{CH}_{3} \mathrm{CH}_{2} \mathrm{O}\right), 2.91\left(\mathrm{~d},{ }^{2} \mathrm{~J}_{\mathrm{PH}}=21.7,2 \mathrm{H}, \mathrm{PC}_{2}\right), 3.84\left(\mathrm{p},{ }^{3} \mathrm{~J}_{\mathrm{HH}}={ }^{3} J_{\mathrm{PH}}=7.0,4 \mathrm{H}, \mathrm{CH}_{3} \mathrm{CH}_{2} \mathrm{O}\right)$, $6.99\left(\mathrm{dd},{ }^{3} \mathrm{~J}_{\mathrm{HH}}=8.4,{ }^{4} \mathrm{~J}_{\mathrm{PH}}=2.5,2 \mathrm{H}, \underline{\mathrm{H}}_{\mathrm{ortho}}\right.$ of $\left.\mathrm{C}_{6} \mathrm{H}_{4}\right), 7.24\left(\mathrm{~d},{ }^{3} \mathrm{~J}_{\mathrm{HH}}=8.4,2 \mathrm{H}, \underline{\mathrm{H}}_{\text {meta }}\right.$ of $\left.\mathrm{C}_{6} \mathrm{H}_{4}\right) . /{ }^{13} \mathrm{C}-\mathrm{NMR} 16.6$ $\left(\mathrm{d},{ }^{3} J_{\mathrm{PC}}=6.0, \underline{\mathrm{CH}}_{3} \mathrm{CH}_{2} \mathrm{O}\right), 33.4\left(\mathrm{~d},{ }^{1} J_{\mathrm{PC}}=138.6, \mathrm{PCH}_{2}\right), 62.4\left(\mathrm{~d},{ }^{2} J_{\mathrm{PC}}=7.3, \mathrm{CH}_{3} \underline{\mathrm{CH}}_{2} \mathrm{O}\right), 121.0\left(\mathrm{~d},{ }^{5} J_{\mathrm{PC}}=4.6\right.$, $\underline{\mathrm{C}}_{\text {para }}$ of $\left.\mathrm{C}_{6} \mathrm{H}_{4}\right), 131.1\left(\mathrm{~d},{ }^{2} J_{\mathrm{PC}}=9.2, \underline{\mathrm{C}}_{\mathrm{ipso}}\right.$ of $\left.\mathrm{C}_{6} \mathrm{H}_{4}\right), 131.7\left(\mathrm{~d},{ }^{3} \mathrm{~J}_{\mathrm{PC}}=6.6, \underline{\mathrm{C}}_{\text {ortho }}\right.$ of $\left.\mathrm{C}_{6} \mathrm{H}_{4}\right), 131.8$ (s, $\underline{\mathrm{C}}_{\text {meta }}$ of $\left.\mathrm{C}_{6} \mathrm{H}_{4}\right)$. / m/z (CI): $307\left(\mathrm{M}+1{ }^{79} \mathrm{Br}, 63\right), 309$ (M+1 $\left.{ }^{81} \mathrm{Br}, 62\right), 324\left(\mathrm{M}+18{ }^{79} \mathrm{Br}, 100\right), 326$ (M+18 $\left.{ }^{81} \mathrm{Br}, 100\right)$.

Diethyl 4-methoxybenzylphosphonate (1i) $\mathrm{C}_{12} \mathrm{H}_{19} \mathrm{O}_{4} \mathrm{P}(\mathrm{M}=258)$ : 31P-NMR +27.3 (s). / 1 H-NMR 1.21 (t, $\left.{ }^{3} J_{\mathrm{HH}}=7.0,6 \mathrm{H}, \mathrm{CH}_{3} \mathrm{CH}_{2} \mathrm{O}\right), 3.06\left(\mathrm{~d},{ }^{2} J_{\mathrm{PH}}=21.1,2 \mathrm{H}, \mathrm{PC}_{2}\right), 3.70\left(\mathrm{~s}, 3 \mathrm{H}, \mathrm{OC}_{3}\right), 3.98\left(\mathrm{p},{ }^{3} J_{\mathrm{HH}}={ }^{3} J_{\mathrm{PH}}=7.1\right.$, $\left.4 \mathrm{H}, \mathrm{CH}_{3} \mathrm{CH}_{2} \mathrm{O}\right), 6.82\left(\mathrm{~d},{ }^{3} \mathrm{~J}_{\mathrm{HH}}=8.1,2 \mathrm{H}, \underline{\mathrm{H}}_{\text {meta }}\right.$ of $\left.\mathrm{C}_{6} \mathrm{H}_{4}\right), 7.21\left(\mathrm{dd},{ }^{3} J_{\mathrm{HH}}=8.7,{ }^{4} J_{\mathrm{PH}}=2.4,2 \mathrm{H}, \underline{\mathrm{H}}_{\mathrm{ortho}}\right.$ of $\left.\mathrm{C}_{6} \mathrm{H}_{4}\right)$. / 13C-NMR $15.7\left(\mathrm{~d},{ }^{3} J_{\mathrm{PC}}=5.3, \underline{\mathrm{CH}}_{3} \mathrm{CH}_{2} \mathrm{O}\right), 31.9\left(\mathrm{~d},{ }^{1} J_{\mathrm{PC}}=138.7, \mathrm{PCH}_{2}\right), 54.3\left(\mathrm{~s}, \mathrm{OCH}_{3}\right), 61.2\left(\mathrm{~d},{ }^{2} J_{\mathrm{PC}}=6.6\right.$, $\left.\mathrm{CH}_{3} \underline{\mathrm{CH}}_{2} \mathrm{O}\right), 113.3$ (s, $\underline{\mathrm{C}}_{\text {meta }}$ of $\left.\mathrm{C}_{6} \mathrm{H}_{4}\right), 122.8\left(\mathrm{~d},{ }^{2} J_{\mathrm{PC}}=9.0, \underline{\mathrm{C}}_{\mathrm{ipso}}\right.$ of $\left.\mathrm{C}_{6} \mathrm{H}_{4}\right), 130.1\left(\mathrm{~d},{ }^{3} J_{\mathrm{PC}}=6.3, \underline{\mathrm{C}}_{\text {ortho }}\right.$ of $\left.\mathrm{C}_{6} \mathrm{H}_{4}\right), 158.0\left(\mathrm{~d},{ }^{5} \mathrm{~J}_{\mathrm{PC}}=2.6, \underline{\mathrm{C}}_{\text {para }}\right.$ of $\left.\mathrm{C}_{6} \mathrm{H}_{4}\right) . / \mathbf{m} / \mathbf{z}(\mathrm{CI}): 259(\mathrm{M}+1,100), 276(\mathrm{M}+18,8)$.

General procedure for the synthesis of diethyl $\alpha$-fluorobenzylphosphonates 
A freshly titrated solution of $n$-BuLi (4.69 $\mathrm{ml}$ of $1.6 \mathrm{M}$ solution in hexane; $7.5 \mathrm{mmol}$ ) was added to THF $(20 \mathrm{ml})$ cooled to $-78^{\circ} \mathrm{C}$. A mixture of hexamethyldisilazane (HMDS) (1.33 g; $\left.8.25 \mathrm{mmol}\right)$ and diethyl benzylphosphonate $(2.5 \mathrm{mmol})$ in THF $(20 \mathrm{ml})$ was then slowly added at this temperature via a dropping funnel. Few minutes later, the reaction mixture was allowed to warm slowly to $20^{\circ} \mathrm{C}$ and $\mathrm{Me}_{3} \mathrm{SiCl}(0.3 \mathrm{~g}$; $2.75 \mathrm{mmol})$ in THF $(10 \mathrm{ml})$ was rapidly added. After $15 \mathrm{~min}$, the reaction mixture was cooled to $-90^{\circ} \mathrm{C}$ and the N-fluorobenzenesulfonimide (NFBS) (1.02 g; $3.25 \mathrm{mmol})$ in THF (20 ml) was slowly added. After 15 min at low temperature, the reaction mixture was allowed to warm-up to $0^{\circ} \mathrm{C}$ and treated at this temperature with an excess of $\mathrm{LiOH}$ in $\mathrm{H}_{2} \mathrm{O}(15 \mathrm{ml})$ for $15 \mathrm{~min}$. The organic layer was washed with $1 \mathrm{M} \mathrm{LiOH}$ solution (2 $\mathrm{x} 15 \mathrm{ml})$ and then poured with stirring into a mixture of $3 \mathrm{M} \mathrm{HCl}(20 \mathrm{ml}), \mathrm{CH}_{2} \mathrm{Cl}_{2}$ or $\mathrm{Et}_{2} \mathrm{O}(20 \mathrm{ml})$ and ice (10 g). After two extractions ( $2 \times 20 \mathrm{ml}$ ) of the aqueous layer, the combined organic layer was dried over $\mathrm{MgSO}_{4}$ and evaporated under reduced pressure to afford the desired compounds as the pale-yellow oil. All isolated compounds were pure enough to make further purification useless, but can be chromatographed on a silica column (70-230 mesh) and eluted with hexane:ethyl acetate (70:30).

Diethyl $\alpha$-fluorobenzylphosphonate $(\mathbf{4 a})^{7,17,30,31} \mathrm{C}_{11} \mathrm{H}_{16} \mathrm{O}_{3} \mathrm{FP}(\mathrm{M}=246):{ }^{31} \mathbf{P}-\mathbf{N M R}+13.0\left(\mathrm{~d},{ }^{2} J_{\mathrm{PF}}=85.6\right)$. I 31P-NMR (1 $\mathbf{H}$ coupled) +13.2 $\left(\mathrm{dd},{ }^{2} J_{\mathrm{PF}}=84.8,{ }^{2} J_{\mathrm{PH}}=7.6\right)$. / ${ }^{19} \mathbf{F}-\mathbf{N M R}-200.6\left(\mathrm{~d},{ }^{2} J_{\mathrm{PF}}=85.6\right)$. / 19F-NMR ( ${ }^{1} \mathbf{H}$ coupled) -201.0 (dd, $\left.{ }^{2} J_{\mathrm{PF}}=83.0,{ }^{2} J_{\mathrm{FH}}=44.4\right)$. / ${ }^{1} \mathbf{H}-\mathrm{NMR} 1.30\left(\mathrm{t},{ }^{3} J_{\mathrm{HH}}=7.1,3 \mathrm{H},\left(\mathrm{CH}_{3} \mathrm{CH}_{2} \mathrm{O}\right)_{\mathrm{A}}\right), 1.32(\mathrm{t}$, $\left.{ }^{3} J_{\mathrm{HH}}=7.1,3 \mathrm{H},\left(\mathrm{CH}_{3} \mathrm{CH}_{2} \mathrm{O}\right)_{\mathrm{B}}\right), 4.01-4.24\left(\mathrm{~m}, 4 \mathrm{H}, \mathrm{CH}_{3} \mathrm{CH}_{2} \mathrm{O}\right), 5.75\left(\mathrm{dd},{ }^{2} J_{\mathrm{PH}}=7.9,{ }^{2} J_{\mathrm{FH}}=44.6,1 \mathrm{H}, \mathrm{C} \underline{\mathrm{HF}}\right)$, 7.41-7.54 (m, 5H, $\left.\mathrm{C}_{6} \underline{\mathrm{H}}_{5}\right)$. / 13C-NMR $16.8\left(\mathrm{~d},{ }^{3} J_{\mathrm{PC}}=5.2, \underline{\mathrm{CH}}_{3} \mathrm{CH}_{2} \mathrm{O}\right), 64.1\left(\mathrm{dd},{ }^{2} J_{\mathrm{PC}}=7.0,{ }^{4} J_{\mathrm{FC}}=18.2\right.$, $\left.\mathrm{CH}_{3} \underline{\mathrm{CH}}_{2} \mathrm{O}\right), 89.9\left(\mathrm{dd},{ }^{1} J_{\mathrm{PC}}=170.5,{ }^{1} J_{\mathrm{FC}}=184.2, \underline{\mathrm{CHF}}\right), 127.3\left(\mathrm{t},{ }^{3} J_{\mathrm{PC}}={ }^{3} J_{\mathrm{FC}}=6.0, \underline{\mathrm{C}}_{\text {ortho }}\right.$ of $\left.\mathrm{C}_{6} \mathrm{H}_{5}\right), 129.0(\mathrm{~d}$, ${ }^{4} J_{\mathrm{FC}}=2.4, \underline{\mathrm{C}}_{\text {meta }}$ of $\left.\mathrm{C}_{6} \mathrm{H}_{5}\right), 129.7\left(\mathrm{~s}, \underline{\mathrm{C}}_{\text {para }}\right.$ of $\left.\mathrm{C}_{6} \mathrm{H}_{5}\right), 133.4\left(\mathrm{~d},{ }^{2} J_{\mathrm{FC}}=18.4, \underline{\mathrm{C}}_{\text {ipso }}\right.$ of $\left.\mathrm{C}_{6} \mathrm{H}_{5}\right) . / \mathbf{m} / \mathbf{z}(\mathrm{CI}): 247$ $(\mathrm{M}+1,100), 264(\mathrm{M}+18,44)$.

Diethyl $\alpha$-fluoro-2-fluorobenzylphosphonate (4b) $\mathrm{C}_{11} \mathrm{H}_{15} \mathrm{O}_{3} \mathrm{~F}_{2} \mathrm{P}(\mathrm{M}=264)$ : 31P-NMR +12.6 (dd, ${ }^{2} J_{\mathrm{PF}}=88.6$, $\left.{ }^{4} J_{\mathrm{PF}}=5.2\right)$. / 31P-NMR (1 $\mathbf{H}$ coupled $)+12.6\left(\mathrm{dt},{ }^{2} J_{\mathrm{PF}}=88.9,{ }^{4} J_{\mathrm{PF}}={ }^{2} J_{\mathrm{PH}}=6.9\right)$. / ${ }^{19} \mathbf{F}-\mathbf{N M R}-117.4\left(\mathrm{~s}, \mathrm{C}_{6} \mathrm{H}_{4} \mathrm{~F}\right),-$ $204.0\left(\mathrm{~d},{ }^{2} J_{\mathrm{PF}}=90.4\right)$. / 19F-NMR (1 $\mathbf{H}$ coupled) $-117.4\left(\mathrm{~s}, \mathrm{C}_{6} \mathrm{H}_{4} \mathrm{~F}\right),-204.0\left(\mathrm{dd},{ }^{2} J_{\mathrm{PF}}=88.2,{ }^{2} J_{\mathrm{FH}}=45.0, \mathrm{CHF}\right) . /$ 1H-NMR $1.33\left(\mathrm{t},{ }^{3} \mathrm{~J}_{\mathrm{HH}}=7.0,3 \mathrm{H},\left(\mathrm{CH}_{3} \mathrm{CH}_{2} \mathrm{O}\right)_{\mathrm{A}}\right), 1.41\left(\mathrm{t},{ }^{3} \mathrm{~J}_{\mathrm{HH}}=7.0,3 \mathrm{H},\left(\mathrm{CH}_{3} \mathrm{CH}_{2} \mathrm{O}\right)_{\mathrm{B}}\right), 4.03-4.34(\mathrm{~m}, 4 \mathrm{H}$, $\left.\mathrm{CH}_{3} \underline{\mathrm{C}}_{2} \mathrm{O}\right), 6.10\left(\mathrm{dd},{ }^{2} J_{\mathrm{PH}}=8.0,{ }^{2} J_{\mathrm{FH}}=44.2,1 \mathrm{H}, \mathrm{C} \underline{\mathrm{HF}}\right), 7.11-7.77\left(\mathrm{~m}, 4 \mathrm{H}, \mathrm{C}_{6} \underline{\mathrm{H}}_{4}\right) . /$ 13C-NMR $17.0(\mathrm{t}$, $\left.{ }^{3} J_{\mathrm{PC}}={ }^{5} J_{\mathrm{FC}}=5.0, \quad \underline{\mathrm{CH}}_{3} \mathrm{CH}_{2} \mathrm{O}\right), 64.3\left(\mathrm{dd},{ }^{2} J_{\mathrm{PC}}=6.7,{ }^{4} J_{\mathrm{FC}}=12.7, \quad \mathrm{CH}_{3} \underline{C H}_{2} \mathrm{O}\right), 83.5 \quad\left(\mathrm{ddd},{ }^{1} J_{\mathrm{PC}}=177.0\right.$, $\left.{ }^{1} J_{\mathrm{FC}}=180.1,{ }^{3} J_{\mathrm{FC}}=3.0, \underline{\mathrm{CHF}}\right), 116.0\left(\mathrm{~d},{ }^{2} J_{\mathrm{FC}}=21.4, \underline{\mathrm{C}}_{\text {meta }}\right.$ of $\left.\mathrm{C}_{6} \mathrm{H}_{4}\right), 121.3\left(\mathrm{dd},{ }^{2} J_{\mathrm{FC}}=19.9,{ }^{2} J_{\mathrm{FC}}=12.2, \underline{\mathrm{C}}_{\mathrm{ipso}}\right.$ of $\left.\mathrm{C}_{6} \mathrm{H}_{4}\right), 125.1$ (s, $\underline{\mathrm{C}}_{\text {ortho' }}$ of $\left.\mathrm{C}_{6} \mathrm{H}_{4}\right), 129.7$ (s, $\underline{\mathrm{C}}_{\text {meta' }}$ of $\left.\mathrm{C}_{6} \mathrm{H}_{4}\right), 131.7\left(\mathrm{~d},{ }^{3} J_{\mathrm{FC}}=8.0, \underline{\mathrm{C}}_{\text {para }}\right.$ of $\left.\mathrm{C}_{6} \mathrm{H}_{4}\right), 160.3$ (dt, ${ }^{1} J_{\mathrm{FC}}=248.7,{ }^{3} J_{\mathrm{PC}}={ }^{3} J_{\mathrm{FC}}=6.1, \underline{\mathrm{C}}_{\mathrm{ortho}}$ of $\left.\mathrm{C}_{6} \mathrm{H}_{4}\right) . / \mathbf{m} / \mathbf{z}(\mathrm{CI}): 265(\mathrm{M}+1,100), 282(\mathrm{M}+18,33)$.

Diethyl $\alpha$-fluoro-3-methylbenzylphosphonate (4c) $\mathrm{C}_{12} \mathrm{H}_{18} \mathrm{O}_{3} \mathrm{FP}(\mathrm{M}=260):$ 31P-NMR $+15.5\left(\mathrm{~d},{ }^{2} J_{\mathrm{PF}}=85.9\right)$. / ${ }^{19}$ F-NMR -198.4 (d, $\left.{ }^{2} J_{\mathrm{PF}}=86.1\right)$. / ${ }^{19} \mathbf{F}-\mathbf{N M R}\left({ }^{1} \mathbf{H}\right.$ coupled) -198.4 (dd, $\left.{ }^{2} J_{\mathrm{PF}}=86.0,{ }^{2} J_{\mathrm{FH}}=45.0\right)$. / 1 H-NMR $1.32\left(\mathrm{t},{ }^{3} J_{\mathrm{HH}}=7.0,3 \mathrm{H},\left(\mathrm{CH}_{3} \mathrm{CH}_{2} \mathrm{O}\right)_{\mathrm{A}}\right), 1.34\left(\mathrm{t},{ }^{3} J_{\mathrm{HH}}=7.0,3 \mathrm{H},\left(\mathrm{C}_{3} \mathrm{CH}_{2} \mathrm{O}\right)_{\mathrm{B}}\right), 2.41\left(\mathrm{~s}, 3 \mathrm{H}, \mathrm{C}_{6} \mathrm{H}_{4} \mathrm{C}_{3}\right), 4.04-$ $4.25\left(\mathrm{~m}, 4 \mathrm{H}, \mathrm{CH}_{3} \underline{\mathrm{C}}_{2} \mathrm{O}\right), 5.75\left(\mathrm{dd},{ }^{2} \mathrm{~J}_{\mathrm{PH}}=7.5,{ }^{2} J_{\mathrm{FH}}=44.7,1 \mathrm{H}, \mathrm{C} \underline{\mathrm{HF}}\right), 7.24-7.64\left(\mathrm{~m}, 4 \mathrm{H}, \mathrm{C}_{6} \underline{\mathrm{H}}_{4}\right) . /{ }^{13} \mathrm{C}-\mathbf{N M R}$ $16.5\left(\mathrm{~d},{ }^{3} J_{\mathrm{PC}}=5.5, \underline{\mathrm{CH}}_{3} \mathrm{CH}_{2} \mathrm{O}\right), 21.6\left(\mathrm{~s}, \mathrm{C}_{6} \mathrm{H}_{4} \underline{\mathrm{CH}}_{3}\right), 64.0\left(\mathrm{dd},{ }^{2} J_{\mathrm{PC}}=7.3,{ }^{4} J_{\mathrm{FC}}=19.2, \mathrm{CH}_{3} \underline{\mathrm{CH}}_{2} \mathrm{O}\right), 89.6(\mathrm{dd}$, $\left.{ }^{1} J_{\mathrm{PC}}=170.0,{ }^{1} J_{\mathrm{FC}}=183.7, \underline{\mathrm{CHF}}\right), 124.1\left(\mathrm{~d},{ }^{3} J_{\mathrm{FC}}=6.0, \underline{\mathrm{C}}_{\text {ortho }}\right.$ of $\left.\mathrm{C}_{6} \mathrm{H}_{4}\right), 124.3\left(\mathrm{~d},{ }^{3} J_{\mathrm{FC}}=6.0, \underline{\mathrm{C}}_{\text {ortho }}\right.$ of $\left.\mathrm{C}_{6} \mathrm{H}_{4}\right)$, $128.6\left(\mathrm{~d},{ }^{5} J_{\mathrm{FC}}=2.1, \underline{\mathrm{C}}_{\text {para }}\right.$ of $\left.\mathrm{C}_{6} \mathrm{H}_{4}\right), 130.3\left(\mathrm{~s}, \underline{\mathrm{C}}_{\text {meta' }}\right.$ of $\left.\mathrm{C}_{6} \mathrm{H}_{4}\right), 132.8\left(\mathrm{~d},{ }^{2} J_{\mathrm{FC}}=18.6, \underline{\mathrm{C}}_{\mathrm{ipso}}\right.$ of $\left.\mathrm{C}_{6} \mathrm{H}_{4}\right), 138.5(\mathrm{~s}$, $\underline{\mathrm{C}}_{\text {meta }}$ of $\left.\mathrm{C}_{6} \mathrm{H}_{4}\right) . / \mathbf{m} / \mathbf{z}(\mathrm{CI}): 261(\mathrm{M}+1,100), 278(\mathrm{M}+18,34)$. 
Diethyl $\alpha$-fluoro-4-methylbenzylphosphonate (4d) ${ }^{7} \mathrm{C}_{12} \mathrm{H}_{18} \mathrm{O}_{3} \mathrm{FP}(\mathrm{M}=260):{ }^{31} \mathbf{P}-\mathbf{N M R}+13.5\left(\mathrm{~d},{ }^{2} J_{\mathrm{PF}}=87.7\right)$. / 31P-NMR (1 H coupled) +13.5 (dd, $\left.{ }^{2} J_{\mathrm{PF}}=86.3,{ }^{2} J_{\mathrm{PH}}=6.5\right)$. / 19F-NMR -199.2 (d, $\left.{ }^{2} J_{\mathrm{PF}}=86.9\right)$. / 19F-NMR ( ${ }^{\mathbf{H}}$ coupled) -199.2 (dd, $\left.{ }^{2} J_{\mathrm{PF}}=86.7,{ }^{2} J_{\mathrm{FH}}=45.0\right)$. / ${ }^{\mathbf{1}} \mathbf{H}-\mathrm{NMR} 1.23\left(\mathrm{t},{ }^{3} J_{\mathrm{HH}}=7.0,3 \mathrm{H},\left(\mathrm{C}_{3} \mathrm{CH}_{2} \mathrm{O}\right)_{\mathrm{A}}\right), 1.26(\mathrm{t}$, $\left.{ }^{3} J_{\mathrm{HH}}=7.0,3 \mathrm{H},\left(\mathrm{C}_{3} \mathrm{CH}_{2} \mathrm{O}\right)_{\mathrm{B}}\right), 2.35\left(\mathrm{~s}, 3 \mathrm{H}, \mathrm{C}_{6} \mathrm{H}_{4} \mathrm{C}_{3}\right), 3.97-4.14\left(\mathrm{~m}, 4 \mathrm{H}, \mathrm{CH}_{3} \mathrm{C}_{2} \mathrm{O}\right), 5.70\left(\mathrm{dd},{ }^{2} J_{\mathrm{PH}}=7.5\right.$, $\left.{ }^{2} J_{\mathrm{FH}}=44.4,1 \mathrm{H}, \mathrm{CHF}\right), 7.21\left(\mathrm{~d},{ }^{3} J_{\mathrm{HH}}=8.0,2 \mathrm{H}, \underline{\mathrm{H}}_{\text {meta }}\right.$ of $\left.\mathrm{C}_{6} \mathrm{H}_{4}\right), 7.34\left(\mathrm{~d},{ }^{3} J_{\mathrm{HH}}=8.0,2 \mathrm{H}, \underline{\mathrm{H}}_{\text {ortho }}\right.$ of $\left.\mathrm{C}_{6} \mathrm{H}_{4}\right)$. / ${ }^{13} \mathrm{C}$-NMR $17.0\left(\mathrm{~d},{ }^{3} J_{\mathrm{PC}}=3.3, \underline{\mathrm{CH}}_{3} \mathrm{CH}_{2} \mathrm{O}\right), 21.7\left(\mathrm{~s}, \mathrm{C}_{6} \mathrm{H}_{4} \underline{\mathrm{CH}_{3}}\right), 64.3\left(\mathrm{dd},{ }^{2} J_{\mathrm{PC}}=6.5,{ }^{4} J_{\mathrm{FC}}=15.4, \mathrm{CH}_{3} \underline{\mathrm{CH}_{2} \mathrm{O}}\right)$, $89.6\left(\mathrm{dd},{ }^{1} J_{\mathrm{PC}}=172.4,{ }^{1} J_{\mathrm{FC}}=183.2, \underline{\mathrm{CHF}}\right), 127.7\left(\mathrm{t},{ }^{3} J_{\mathrm{PC}}={ }^{3} J_{\mathrm{FC}}=6.0, \underline{\mathrm{C}}_{\text {ortho }}\right.$ of $\left.\mathrm{C}_{6} \mathrm{H}_{4}\right), 129.9$ (s, $\underline{\mathrm{C}}_{\text {meta }}$ of $\left.\mathrm{C}_{6} \mathrm{H}_{4}\right), 130.4\left(\mathrm{~d},{ }^{2} J_{\mathrm{FC}}=15.0, \underline{\mathrm{C}}_{\mathrm{ipso}}\right.$ of $\left.\mathrm{C}_{6} \mathrm{H}_{4}\right), 140.1\left(\mathrm{~d},{ }^{5} J_{\mathrm{FC}}=3.1, \underline{\mathrm{C}}_{\mathrm{para}}\right.$ of $\left.\mathrm{C}_{6} \mathrm{H}_{4}\right) . / \mathbf{m} / \mathbf{z}(\mathrm{CI}): 261(\mathrm{M}+1,100)$, $278(\mathrm{M}+18,37)$.

Diethyl $\alpha$-fluoro-4-fluorobenzylphosphonate (4e) $\mathrm{C}_{11} \mathrm{H}_{15} \mathrm{O}_{3} \mathrm{~F}_{2} \mathrm{P}(\mathrm{M}=264)$ : 31P-NMR +12.9 (d, $\left.{ }^{2} J_{\mathrm{PF}}=85.6\right)$. / 19F-NMR -112.4 (s, $\left.\mathrm{C}_{6} \mathrm{H}_{4} \mathrm{~F}\right),-199.2\left(\mathrm{~d},{ }^{2} J_{\mathrm{PF}}=85.8\right)$. / 19F-NMR (1 H coupled) -112.4 (s, $\left.\mathrm{C}_{6} \mathrm{H}_{4} \mathrm{~F}\right),-199.1$ (dd, $\left.{ }^{2} J_{\mathrm{PF}}=85.9,{ }^{2} J_{\mathrm{FH}}=45.0, \mathrm{CHF}\right) . /{ }^{\mathbf{1}} \mathbf{H}-\mathrm{NMR} 1.24\left(\mathrm{t},{ }^{3} J_{\mathrm{HH}}=7.0,6 \mathrm{H}, \mathrm{CH}_{3} \mathrm{CH}_{2} \mathrm{O}\right), 3.94-4.16\left(\mathrm{~m}, 4 \mathrm{H}, \mathrm{CH}_{3} \mathrm{CH}_{2} \mathrm{O}\right)$, $5.62\left(\mathrm{dd},{ }^{2} J_{\mathrm{PH}}=7.5,{ }^{2} J_{\mathrm{FH}}=44.4,1 \mathrm{H}, \mathrm{C} \underline{\mathrm{HF}}\right), 7.06\left(\mathrm{t},{ }^{3} J_{\mathrm{HH}}={ }^{3} J_{\mathrm{FH}}=8.6,2 \mathrm{H}, \underline{\mathrm{H}}_{\text {meta }}\right.$ of $\left.\mathrm{C}_{6} \mathrm{H}_{4}\right), 7.44(\mathrm{t}$, ${ }^{3} J_{\mathrm{HH}}={ }^{4} J_{\mathrm{FH}}=6.3,2 \mathrm{H}, \underline{\mathrm{H}}_{\text {ortho }}$ of $\left.\mathrm{C}_{6} \mathrm{H}_{4}\right) . /{ }^{13} \mathrm{C}-\mathrm{NMR} 17.0\left(\mathrm{~d},{ }^{3} J_{\mathrm{PC}}=3.5, \underline{\mathrm{CH}}_{3} \mathrm{CH}_{2} \mathrm{O}\right), 64.5\left(\mathrm{dd},{ }^{2} J_{\mathrm{PC}}=6.8\right.$, $\left.{ }^{4} J_{\mathrm{FC}}=19.5, \mathrm{CH}_{3} \underline{\mathrm{CH}}_{2} \mathrm{O}\right), 88.7\left(\mathrm{dd},{ }^{1} J_{\mathrm{PC}}=171.5,{ }^{1} J_{\mathrm{FC}}=183.9, \underline{\mathrm{C} H F}\right), 116.2\left(\mathrm{~d},{ }^{2} J_{\mathrm{FC}}=21.0, \underline{\mathrm{C}}_{\mathrm{meta}}\right.$ of $\left.\mathrm{C}_{6} \mathrm{H}_{4}\right)$, $129.4\left(\mathrm{~d},{ }^{2} J_{\mathrm{FC}}=21.3, \underline{\mathrm{C}}_{\text {ipso }}\right.$ of $\left.\mathrm{C}_{6} \mathrm{H}_{4}\right), 129.5\left(\mathrm{~d},{ }^{3} J_{\mathrm{FC}}=4.2, \underline{C}_{\text {ortho }}\right.$ of $\left.\mathrm{C}_{6} \mathrm{H}_{4}\right), 163.8\left(\mathrm{dt},{ }^{1} J_{\mathrm{FC}}=248.7\right.$, ${ }^{5} J_{\mathrm{PC}}={ }^{5} J_{\mathrm{FC}}=3.0, \underline{\mathrm{C}}_{\text {para }}$ of $\left.\mathrm{C}_{6} \mathrm{H}_{4}\right) . / \mathbf{m} / \mathbf{z}(\mathrm{CI}): 265(\mathrm{M}+1,53), 282(\mathrm{M}+18,100)$.

Diethyl $\alpha$-fluoro-4-chlorobenzylphosphonate $(\mathbf{4 f})^{7} \quad \mathrm{C}_{11} \mathrm{H}_{15} \mathrm{O}_{3} \mathrm{FClP} \quad(\mathrm{M}=280.5)$ : $31 \mathbf{P}-\mathbf{N M R}+12.6$ (d, $\left.{ }^{2} J_{\mathrm{PF}}=83.3\right)$. / 31P-NMR $\left({ }^{1} \mathrm{H}\right.$ coupled $)+12.6\left(\mathrm{dd},{ }^{2} J_{\mathrm{PF}}=84.6,{ }^{2} J_{\mathrm{PH}}=7.2\right)$. / 19F-NMR $-201.4\left(\mathrm{~d},{ }^{2} J_{\mathrm{PF}}=83.6\right)$. / 19F-NMR (1 $\mathbf{H}$ coupled) $-201.3\left(\mathrm{dd},{ }^{2} J_{\mathrm{PF}}=83.1,{ }^{2} J_{\mathrm{FH}}=46.6\right) . \quad / \quad \mathbf{1} \mathbf{H}-\mathbf{N M R} 1.22\left(\mathrm{t},{ }^{3} J_{\mathrm{HH}}=7.0,3 \mathrm{H}\right.$, $\left.\left(\mathrm{CH}_{3} \mathrm{CH}_{2} \mathrm{O}\right)_{\mathrm{A}}\right), 1.24\left(\mathrm{t},{ }^{3} \mathrm{~J}_{\mathrm{HH}}=7.0,3 \mathrm{H},\left(\mathrm{CH}_{3} \mathrm{CH}_{2} \mathrm{O}\right)_{\mathrm{B}}\right), 3.95-4.16\left(\mathrm{~m}, 4 \mathrm{H}, \mathrm{CH}_{3} \underline{\mathrm{H}}_{2} \mathrm{O}\right), 5.70\left(\mathrm{dd},{ }^{2} J_{\mathrm{PH}}=7.3\right.$, $\left.{ }^{2} J_{\mathrm{FH}}=44.5,1 \mathrm{H}, \mathrm{C} \underline{\mathrm{HF}}\right), 6.95-7.34\left(\mathrm{~m}, 4 \mathrm{H}, \mathrm{C}_{6} \underline{\mathrm{H}}_{4}\right)$. / ${ }^{13} \mathrm{C}-\mathrm{NMR} 17.0\left(\mathrm{~d},{ }^{3} J_{\mathrm{PC}}=5.5, \underline{\mathrm{CH}}_{3} \mathrm{CH}_{2} \mathrm{O}\right), 64.3(\mathrm{dd}$, $\left.{ }^{2} J_{\mathrm{PC}}=7.1,{ }^{4} J_{\mathrm{FC}}=20.1, \mathrm{CH}_{3} \underline{\mathrm{CH}_{2} \mathrm{O}}\right), 88.9\left(\mathrm{dd},{ }^{1} J_{\mathrm{PC}}=172.9,{ }^{1} J_{\mathrm{FC}}=184.0, \underline{\mathrm{CHF}}\right), 128.7\left(\mathrm{t},{ }^{3} J_{\mathrm{PC}}={ }^{3} J_{\mathrm{FC}}=6.0, \underline{\mathrm{C}}_{\mathrm{ortho}}\right.$ of $\left.\mathrm{C}_{6} \mathrm{H}_{4}\right), 129.3$ ( $\mathrm{s}, \underline{\mathrm{C}}_{\text {meta }}$ of $\left.\mathrm{C}_{6} \mathrm{H}_{4}\right), 132.1\left(\mathrm{~d},{ }^{2} J_{\mathrm{FC}}=19.5, \underline{\mathrm{C}}_{\mathrm{ipso}}\right.$ of $\left.\mathrm{C}_{6} \mathrm{H}_{4}\right), 135.7\left(\mathrm{t},{ }^{5} J_{\mathrm{PC}}={ }^{5} J_{\mathrm{FC}}=2.9, \underline{\mathrm{C}}_{\mathrm{para}}\right.$ of $\left.\mathrm{C}_{6} \mathrm{H}_{4}\right) . / \mathbf{m} / \mathbf{z}(\mathrm{CI}): 281\left(\mathrm{M}+1{ }^{35} \mathrm{Cl}, 100\right), 283\left(\mathrm{M}+1{ }^{37} \mathrm{Cl}, 34\right), 298\left(\mathrm{M}+18{ }^{35} \mathrm{Cl}, 97\right), 300\left(\mathrm{M}+18{ }^{37} \mathrm{Cl}, 32\right)$.

Diethyl $\alpha$-fluoro-4-bromobenzylphosphonate (4g) $\quad \mathrm{C}_{11} \mathrm{H}_{15} \mathrm{O}_{3} \mathrm{FBrP} \quad(\mathrm{M}=325) \quad: \quad 31 \mathbf{P}-\mathbf{N M R}+14.4 \quad(\mathrm{~d}$, $\left.{ }^{2} J_{\mathrm{PF}}=85.1\right)$. / 31P-NMR $\left({ }^{1} \mathrm{H}\right.$ coupled $)+14.4\left(\mathrm{dd},{ }^{2} J_{\mathrm{PF}}=83.7,{ }^{2} J_{\mathrm{PH}}=7.6\right)$. / 19F-NMR $-199.7\left(\mathrm{~d},{ }^{2} J_{\mathrm{PF}}=85.4\right)$. / ${ }^{19} \mathbf{F}-\mathbf{N M R}\left({ }^{1} \mathrm{H}\right.$ coupled) $-199.8\left(\mathrm{dd},{ }^{2} J_{\mathrm{PF}}=83.2,{ }^{2} J_{\mathrm{FH}}=45.8\right) . / 1 \mathbf{H}-\mathbf{N M R} 1.21 \quad\left(\mathrm{t},{ }^{3} J_{\mathrm{HH}}=7.0,3 \mathrm{H}\right.$, $\left.\left(\mathrm{C}_{3} \mathrm{CH}_{2} \mathrm{O}\right)_{\mathrm{A}}\right), 1.24\left(\mathrm{t},{ }^{3} J_{\mathrm{HH}}=7.0,3 \mathrm{H},\left(\mathrm{CH}_{3} \mathrm{CH}_{2} \mathrm{O}\right)_{\mathrm{B}}\right), 3.90-4.16\left(\mathrm{~m}, 4 \mathrm{H}, \mathrm{CH}_{3} \underline{\mathrm{H}}_{2} \mathrm{O}\right), 5.77\left(\mathrm{dd},{ }^{2} J_{\mathrm{PH}}=8.0\right.$, $\left.{ }^{2} J_{\mathrm{FH}}=44.6,1 \mathrm{H}, \mathrm{C} \underline{\mathrm{HF}}\right), 7.30\left(\mathrm{dd},{ }^{3} J_{\mathrm{HH}}=8.6,{ }^{4} J_{\mathrm{FH}}=1.8,2 \mathrm{H}, \underline{\mathrm{H}}_{\mathrm{ortho}}\right.$ of $\left.\mathrm{C}_{6} \mathrm{H}_{4}\right), 7.48\left(\mathrm{~d},{ }^{3} J_{\mathrm{HH}}=8.6,2 \mathrm{H}, \underline{\mathrm{H}}_{\text {meta }}\right.$ of

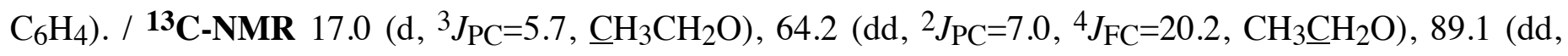
$\left.{ }^{1} J_{\mathrm{PC}}=171.0,{ }^{1} J_{\mathrm{FC}}=184.6, \underline{\mathrm{CHF}}\right), 123.9\left(\mathrm{t},{ }^{5} J_{\mathrm{PC}}=5 J_{\mathrm{FC}}=3.0, \underline{\mathrm{C}}_{\mathrm{para}}\right.$ of $\left.\mathrm{C}_{6} \mathrm{H}_{4}\right), 128.9\left(\mathrm{t},{ }^{3} J_{\mathrm{PC}}={ }^{3} J_{\mathrm{FC}}=6.7, \underline{\mathrm{C}}_{\mathrm{ortho}}\right.$ of $\left.\mathrm{C}_{6} \mathrm{H}_{4}\right), 132.2\left(\mathrm{~d},{ }^{4} J_{\mathrm{FC}}=2.7, \underline{\mathrm{C}}_{\text {meta }}\right.$ of $\left.\mathrm{C}_{6} \mathrm{H}_{4}\right), 132.6\left(\mathrm{~d},{ }^{2} J_{\mathrm{FC}}=19.0, \underline{\mathrm{C}}_{\text {ipso }}\right.$ of $\left.\mathrm{C}_{6} \mathrm{H}_{4}\right) . / \mathbf{m} / \mathbf{z}(\mathrm{CI}): 325\left(\mathrm{M}+1{ }^{79} \mathrm{Br}\right.$, 100), $327\left(\mathrm{M}+1{ }^{81} \mathrm{Br}, 100\right), 342\left(\mathrm{M}+18^{79} \mathrm{Br}, 44\right), 344\left(\mathrm{M}+18^{81} \mathrm{Br}, 44\right)$.

Diethyl $\alpha$-fluoro-4-methoxybenzylphosphonate (4h) $\mathrm{C}_{12} \mathrm{H}_{18} \mathrm{O}_{4} \mathrm{FP}(\mathrm{M}=276):{ }^{31} \mathbf{P}-\mathbf{N M R}+15.8\left(\mathrm{~d},{ }^{2} J_{\mathrm{PF}}=91.3\right)$. / 31P-NMR (1 $\mathbf{H}$ coupled) +15.8 (dd, $\left.{ }^{2} J_{\mathrm{PF}}=91.1,{ }^{2} J_{\mathrm{PH}}=5.4\right)$. / 19F-NMR -192.5 (d, $\left.{ }^{2} J_{\mathrm{PF}}=90.3\right)$. / 19F-NMR (1 ${ }^{1}$ coupled) -192.5 (dd, $\left.{ }^{2} J_{\mathrm{PF}}=90.8,{ }^{2} J_{\mathrm{FH}}=42.4\right)$. / ${ }^{1} \mathbf{H}-\mathrm{NMR} 1.25\left(\mathrm{t},{ }^{3} J_{\mathrm{HH}}=7.1,3 \mathrm{H},\left(\mathrm{C}_{3} \mathrm{CH}_{2} \mathrm{O}\right)_{\mathrm{A}}\right), 1.30(\mathrm{t}$, $\left.{ }^{3} J_{\mathrm{HH}}=7.1,3 \mathrm{H},\left(\mathrm{CH}_{3} \mathrm{CH}_{2} \mathrm{O}\right)_{\mathrm{B}}\right), 3.70-4.19\left(\mathrm{~m}, 4 \mathrm{H}, \mathrm{CH}_{3} \underline{\mathrm{CH}}_{2} \mathrm{O}\right), 3.80\left(\mathrm{~s}, 3 \mathrm{H}, \mathrm{OCH}_{3}\right), 5.66\left(\mathrm{dd},{ }^{2} J_{\mathrm{PH}}=7.0\right.$, 
$\left.{ }^{2} J_{\mathrm{FH}}=44.4,1 \mathrm{H}, \mathrm{C} \underline{\mathrm{HF}}\right), 6.93\left(\mathrm{~d},{ }^{3} J_{\mathrm{HH}}=8.6,2 \mathrm{H}, \underline{\mathrm{H}}_{\text {meta }}\right.$ of $\left.\mathrm{C}_{6} \mathrm{H}_{4}\right), 7.91\left(\mathrm{~d},{ }^{3} J_{\mathrm{HH}}=8.6,2 \mathrm{H}, \underline{\mathrm{H}}_{\mathrm{ortho}}\right.$ of $\left.\mathrm{C}_{6} \mathrm{H}_{4}\right) . /$ ${ }^{13} \mathbf{C}$-NMR $16.8\left(\mathrm{dd},{ }^{3} J_{\mathrm{FC}}=3.1,{ }^{3} J_{\mathrm{PC}}=5.6, \underline{\mathrm{CH}}_{3} \mathrm{CH}_{2} \mathrm{O}\right), 55.8\left(\mathrm{~s}, \mathrm{OC}_{3}\right), 64.3\left(\mathrm{dd},{ }^{2} J_{\mathrm{PC}}=7.1,{ }^{4} J_{\mathrm{FC}}=14.0\right.$, $\left.\mathrm{CH}_{3} \underline{\mathrm{CH}}_{2} \mathrm{O}\right), 89.5\left(\mathrm{dd},{ }^{1} J_{\mathrm{PC}}=173.9,{ }^{1} J_{\mathrm{FC}}=183.0, \underline{\mathrm{CHF}}\right), 114.5\left(\mathrm{~s}, \underline{\mathrm{C}}_{\text {meta }}\right.$ of $\left.\mathrm{C}_{6} \mathrm{H}_{4}\right), 125.1\left(\mathrm{~d},{ }^{2} J_{\mathrm{FC}}=19.5, \underline{\mathrm{C}}_{\mathrm{ipso}}\right.$ of $\left.\mathrm{C}_{6} \mathrm{H}_{4}\right), 129.1$ (d, ${ }^{3} J_{\mathrm{FC}}=10.7, \underline{\mathrm{C}}_{\text {ortho }}$ of $\left.\mathrm{C}_{6} \mathrm{H}_{4}\right), 161.0\left(\mathrm{~s}, \underline{\mathrm{C}}_{\text {para }}\right.$ of $\left.\mathrm{C}_{6} \mathrm{H}_{4}\right) . / \mathbf{m} / \mathbf{z}(\mathrm{CI}): 277(\mathrm{M}+1,100), 294$ $(\mathrm{M}+18,43)$.

General procedure for the synthesis of diethyl $\alpha$-chloro, bromo and iodo-benzylphosphonates

A freshly titrated solution of $n$-BuLi (18.75 $\mathrm{ml}$ of $1.6 \mathrm{M}$ solution in hexane; $30 \mathrm{mmol})$ was added to THF (20 ml) cooled to $-78^{\circ} \mathrm{C}$. A mixture of hexamethyldisilazane (HMDS) (5.15 g; $\left.32 \mathrm{mmol}\right)$ and diethyl benzylphosphonate $(10 \mathrm{mmol})$ in THF $(20 \mathrm{ml})$ was slowly added at this temperature via a dropping funnel. Few minutes later, the reaction mixture was allowed to warm slowly to $20^{\circ} \mathrm{C}$ and $\mathrm{Me}_{3} \mathrm{SiCl}(1.3 \mathrm{~g} ; 12 \mathrm{mmol})$ in THF $(10 \mathrm{ml})$ was rapidly added. After $15 \mathrm{~min}$, the reaction mixture was cooled to $-78^{\circ} \mathrm{C}$ and the halogenating agent $(11 \mathrm{mmol})$ in THF $(20 \mathrm{ml})$ was slowly added. After $15 \mathrm{~min}$, the reaction mixture was allowed to warm-up to $0^{\circ} \mathrm{C}$ and then treated with an excess of EtOLi in EtOH. After 15 min the solution was poured with stirring into a mixture of $3 \mathrm{M} \mathrm{HCl}(20 \mathrm{ml}), \mathrm{CH}_{2} \mathrm{Cl}_{2}(20 \mathrm{ml})$ and ice $(10 \mathrm{~g})$ and the aqueous layer was extracted with $\mathrm{CH}_{2} \mathrm{Cl}_{2}(2 \times 20 \mathrm{ml})$. The combined organic extracts are dried over $\mathrm{MgSO}_{4}$ and evaporated under reduced pressure to afford the desired products as the pale-yellow oil for the chloro and bromo compound and as the reddish-brown oil for the iodo compound. All isolated products were pure enough to make further purification useless. The chloro and bromo derivatives can be distilled under reduced pressure with a bulb-to-bulb apparatus or chromatographed on a silica column (70-230 mesh) and eluted with hexane:ethyl acetate (70:30).

Diethyl $\alpha$-chlorobenzylphosphonate $(\mathbf{5 a})^{30} \mathrm{C}_{11} \mathrm{H}_{16} \mathrm{O}_{3} \mathrm{ClP}(\mathrm{M}=262.5)$ : 31P-NMR +18.2 (s). / 1 H-NMR 1.14 $\left(\mathrm{t},{ }^{3} J_{\mathrm{HH}}=7.1,3 \mathrm{H},\left(\mathrm{C}_{3} \mathrm{CH}_{2} \mathrm{O}\right)_{\mathrm{A}}\right), 1.29\left(\mathrm{t},{ }^{3} \mathrm{~J}_{\mathrm{HH}}=7.0,3 \mathrm{H},\left(\mathrm{CH}_{3} \mathrm{CH}_{2} \mathrm{O}\right)_{\mathrm{B}}\right), 3.75-4.05\left(\mathrm{~m}, 2 \mathrm{H},\left(\mathrm{CH}_{3} \mathrm{C}_{2} \mathrm{O}\right)_{\mathrm{B}}\right)$, $4.15\left(\mathrm{p},{ }^{3} J_{\mathrm{HH}}={ }^{3} J_{\mathrm{PH}}=7.1,2 \mathrm{H},\left(\mathrm{CH}_{3} \underline{\mathrm{H}}_{2} \mathrm{O}\right) \mathrm{A}\right), 4.87\left(\mathrm{~d},{ }^{2} J_{\mathrm{PH}}=14.1,1 \mathrm{H}, \mathrm{C} \underline{\mathrm{HCl}}\right), 7.26-7.49\left(\mathrm{~m}, 3 \mathrm{H}, \underline{\mathrm{H}}_{\mathrm{meta}}\right.$,para of $\left.\mathrm{C}_{6} \mathrm{H}_{5}\right), 7.47-7.56\left(\mathrm{~m}, 2 \mathrm{H}, \underline{\mathrm{H}}_{\mathrm{ortho}}\right.$ of $\left.\mathrm{C}_{6} \mathrm{H}_{5}\right) . /{ }^{13} \mathrm{C}-\mathrm{NMR} 15.9\left(\mathrm{~d},{ }^{3} J_{\mathrm{PC}}=5.3,\left(\underline{\mathrm{CH}}_{3} \mathrm{CH}_{2} \mathrm{O}\right) \mathrm{A}\right), 16.1\left(\mathrm{~d},{ }^{3} J_{\mathrm{PC}}=4.7\right.$,

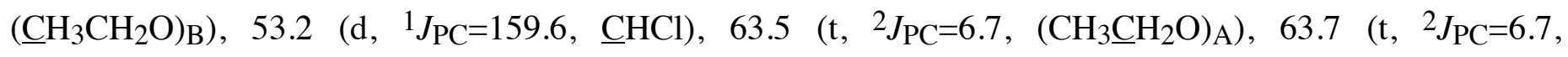
$\left.\left(\mathrm{CH}_{3} \underline{\mathrm{CH}}_{2} \mathrm{O}\right)_{\mathrm{B}}\right), 128.2\left(\mathrm{~s}, \underline{\mathrm{C}}_{\text {meta }}\right.$ of $\left.\mathrm{C}_{6} \mathrm{H}_{5}\right), 128.7$ (s, $\underline{\mathrm{C}}_{\text {para }}$ of $\left.\mathrm{C}_{6} \mathrm{H}_{5}\right), 128.8$ (s, $\underline{\mathrm{C}}_{\text {ortho }}$ of $\left.\mathrm{C}_{6} \mathrm{H}_{5}\right), 134.0$ (s, $\underline{\mathrm{C}}_{\mathrm{ipso}}$ of $\left.\mathrm{C}_{6} \mathrm{H}_{5}\right) . / \mathbf{m} / \mathbf{z}(\mathrm{CI}): 263\left(\mathrm{M}+1{ }^{35} \mathrm{Cl}, 100\right), 265\left(\mathrm{M}+1{ }^{37} \mathrm{Cl}, 34\right), 280\left(\mathrm{M}+18{ }^{35} \mathrm{Cl}, 31\right), 282\left(\mathrm{M}+18{ }^{37} \mathrm{Cl}, 9\right)$.

Diethyl $\alpha$-chloro-2-fluorobenzylphosphonate (5b) $\mathrm{C}_{11} \mathrm{H}_{15} \mathrm{O}_{3} \mathrm{FClP}(\mathrm{M}=280.5):$ 31P-NMR +16.6 (s). / 19F-NMR -113.8 (s, $\left.\mathrm{C}_{6} \mathrm{H}_{4} \underline{\mathrm{F}}\right)$. / 1 $\mathbf{H}-\mathrm{NMR} 1.04\left(\mathrm{t},{ }^{3} \mathrm{~J}_{\mathrm{HH}}=7.0,3 \mathrm{H},\left(\mathrm{C}_{3} \mathrm{CH}_{2} \mathrm{O}\right)_{\mathrm{A}}\right), 1.19\left(\mathrm{t},{ }^{3} J_{\mathrm{HH}}=7.0,3 \mathrm{H}\right.$, $\left.\left(\mathrm{CH}_{3} \mathrm{CH}_{2} \mathrm{O}\right)_{\mathrm{B}}\right), 3.75-4.04\left(\mathrm{~m}, 2 \mathrm{H},\left(\mathrm{CH}_{3} \mathrm{CH}_{2} \mathrm{O}\right)_{\mathrm{B}}\right), 4.10\left(\mathrm{p},{ }^{3} \mathrm{~J}_{\mathrm{HH}}={ }^{3} \mathrm{~J}_{\mathrm{PH}}=7.0,2 \mathrm{H},\left(\mathrm{CH}_{3} \mathrm{CH}_{2} \mathrm{O}\right)_{\mathrm{A}}\right), 5.20(\mathrm{~d}$, $\left.{ }^{2} J_{\mathrm{PH}}=14.5,1 \mathrm{H}, \mathrm{C} \underline{\mathrm{HCl}}\right), 6.86-7.24\left(\mathrm{~m}, 3 \mathrm{H}, \underline{\mathrm{H}}_{\mathrm{ortho}}\right.$,meta,meta' of $\left.\mathrm{C}_{6} \mathrm{H}_{4}\right), 7.65\left(\mathrm{dt},{ }^{3} J_{\mathrm{HH}}={ }^{4} J_{\mathrm{FH}}=7.6,{ }^{4} J_{\mathrm{HH}}=1.8\right.$, $1 \mathrm{H}, \underline{\mathrm{H}}_{\text {para }}$ of $\left.\mathrm{C}_{6} \mathrm{H}_{4}\right) . /{ }^{13} \mathrm{C}-\mathrm{NMR} 16.5\left(\mathrm{~d},{ }^{3} \mathrm{~J}_{\mathrm{PC}}=5.8,\left(\mathrm{CH}_{3} \mathrm{CH}_{2} \mathrm{O}\right)_{\mathrm{A}}\right), 16.7\left(\mathrm{~d},{ }^{3} \mathrm{~J}_{\mathrm{PC}}=5.3,\left(\underline{\mathrm{CH}}_{3} \mathrm{CH}_{2} \mathrm{O}\right)_{\mathrm{B}}\right), 45.5$ $\left(\mathrm{dd},{ }^{1} J_{\mathrm{PC}}=163.3,{ }^{3} J_{\mathrm{FC}}=4.3, \underline{\mathrm{CHCl}}\right), 64.3\left(\mathrm{~d},{ }^{2} J_{\mathrm{PC}}=7.4,\left(\mathrm{CH}_{3} \underline{\mathrm{CH}}_{2} \mathrm{O}\right)_{\mathrm{A}}\right), 64.6\left(\mathrm{~d},{ }^{2} J_{\mathrm{PC}}=6.4,\left(\mathrm{CH}_{3} \underline{\mathrm{CH}}_{2} \mathrm{O}\right)_{\mathrm{B}}\right)$, $115.6\left(\mathrm{~d},{ }^{2} J_{\mathrm{FC}}=21.6, \underline{\mathrm{C}}_{\text {meta }}\right.$ of $\left.\mathrm{C}_{6} \mathrm{H}_{4}\right), 122.3\left(\mathrm{dd},{ }^{2} J_{\mathrm{PC}}=13.1,{ }^{2} J_{\mathrm{FC}}=2.9, \mathrm{C}_{\text {ipso }}\right.$ of $\left.\mathrm{C}_{6} \mathrm{H}_{4}\right), 124.4(\mathrm{t}$, ${ }^{4} J_{\mathrm{PC}}={ }^{4} J_{\mathrm{FC}}=3.0, \underline{\mathrm{C}}_{\text {meta' }}$ of $\left.\mathrm{C}_{6} \mathrm{H}_{4}\right), 131.0\left(\mathrm{~d},{ }^{3} J_{\mathrm{FC}}=2.5, \underline{\mathrm{C}}_{\text {ortho' }}\right.$ of $\left.\mathrm{C}_{6} \mathrm{H}_{4}\right), 131.2\left(\mathrm{~d},{ }^{3} J_{\mathrm{FC}}=4.6, \underline{\mathrm{C}}_{\mathrm{para}}\right.$ of $\left.\mathrm{C}_{6} \mathrm{H}_{4}\right)$, $160.0\left(\mathrm{dd},{ }^{3} J_{\mathrm{PC}}=7.6,{ }^{1} J_{\mathrm{FC}}=248.7, \underline{\mathrm{C}}_{\text {ortho }}\right.$ of $\left.\mathrm{C}_{6} \mathrm{H}_{4}\right) . / \mathbf{~ m} / \mathbf{z}(\mathrm{CI}): 281\left(\mathrm{M}+1{ }^{35} \mathrm{Cl}, 100\right), 283\left(\mathrm{M}+1{ }^{37} \mathrm{Cl}, 30\right), 298$ $\left(\mathrm{M}+18{ }^{35} \mathrm{Cl}, 30\right), 300\left(\mathrm{M}+18{ }^{37} \mathrm{Cl}, 10\right)$. 
Diethyl $\alpha$-chloro-3-methylbenzylphosphonate (5c) $\mathrm{C}_{12} \mathrm{H}_{18} \mathrm{O}_{3} \mathrm{ClP}(\mathrm{M}=276.5)$ : 31P-NMR +17.3 (s). / 1H-NMR $1.22\left(\mathrm{t},{ }^{3} J_{\mathrm{HH}}=7.0,3 \mathrm{H},\left(\mathrm{C}_{3} \mathrm{CH}_{2} \mathrm{O}\right)_{\mathrm{A}}\right), 1.37\left(\mathrm{t},{ }^{3} J_{\mathrm{HH}}=7.1,3 \mathrm{H},\left(\mathrm{C}_{3} \mathrm{CH}_{2} \mathrm{O}\right)_{\mathrm{B}}\right), 2.40(\mathrm{~s}, 3 \mathrm{H}$, $\left.\mathrm{C}_{6} \mathrm{H}_{4} \underline{\mathrm{C}}_{3}\right), 3.86-4.31\left(\mathrm{~m}, 4 \mathrm{H}, \mathrm{CH}_{3} \underline{\mathrm{C}}_{2} \mathrm{O}\right), 4.93\left(\mathrm{~d},{ }^{2} \mathrm{~J}_{\mathrm{PH}}=14.0,1 \mathrm{H}, \mathrm{C} \underline{\mathrm{HCl}}\right), 7.17-7.40\left(\mathrm{~m}, 4 \mathrm{H}, \mathrm{C}_{6} \underline{\mathrm{H}}_{4}\right)$. /

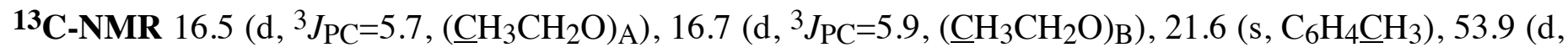
$\left.{ }^{1} J_{\mathrm{PC}}=160.0, \underline{\mathrm{CHCl}}\right), 64.2\left(\mathrm{t},{ }^{2} J_{\mathrm{PC}}=7.7,\left(\mathrm{CH}_{3} \underline{\mathrm{CH}}_{2} \mathrm{O}\right)_{\mathrm{A}}\right), 64.4\left(\mathrm{t},{ }^{2} J_{\mathrm{PC}}=7.7,\left(\mathrm{CH}_{3} \underline{\mathrm{CH}}_{2} \mathrm{O}\right)_{\mathrm{B}}\right), 126.3\left(\mathrm{~d},{ }^{3} J_{\mathrm{PC}}=6.0\right.$,

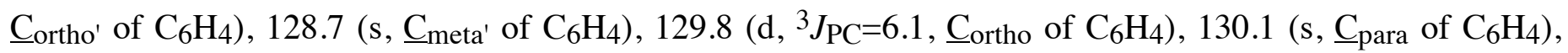
$134.2\left(\mathrm{~d},{ }^{2} \mathrm{~J}_{\mathrm{PC}}=3.0, \underline{\mathrm{C}}_{\text {ipso }}\right.$ of $\left.\mathrm{C}_{6} \mathrm{H}_{4}\right), 138.5\left(\mathrm{~s}, \underline{\mathrm{C}}_{\text {meta }}\right.$ of $\left.\mathrm{C}_{6} \mathrm{H}_{4}\right) . / \mathbf{m} / \mathbf{z}(\mathrm{CI}): 277\left(\mathrm{M}+1{ }^{35} \mathrm{Cl}, 100\right), 279(\mathrm{M}+1$ $\left.{ }^{37} \mathrm{Cl}, 40\right), 294\left(\mathrm{M}+18{ }^{35} \mathrm{Cl}, 27\right), 296\left(\mathrm{M}+18{ }^{37} \mathrm{Cl}, 9\right)$.

Diethyl $\alpha$-chloro-4-methylbenzylphosphonate (5d) $\mathrm{C}_{12} \mathrm{H}_{18} \mathrm{O}_{3} \mathrm{ClP}(\mathrm{M}=276.5)$ : 31P-NMR +17.5 (s). / 1H-NMR $1.21\left(\mathrm{t},{ }^{3} \mathrm{~J}_{\mathrm{HH}}=7.1,3 \mathrm{H},\left(\mathrm{CH}_{3} \mathrm{CH}_{2} \mathrm{O}\right)_{\mathrm{A}}\right), 1.35\left(\mathrm{t},{ }^{3} \mathrm{~J}_{\mathrm{HH}}=7.0,3 \mathrm{H},\left(\mathrm{CH}_{3} \mathrm{CH}_{2} \mathrm{O}\right)_{\mathrm{B}}\right), 2.37(\mathrm{~s}, 3 \mathrm{H}$, $\left.\mathrm{C}_{6} \mathrm{H}_{4} \underline{\mathrm{C}}_{3}\right), 3.90-4.11\left(\mathrm{~m}, 2 \mathrm{H},\left(\mathrm{CH}_{3} \underline{\mathrm{C}}_{2} \mathrm{O}\right)_{\mathrm{B}}\right), 4.21\left(\mathrm{p},{ }^{3} J_{\mathrm{HH}}={ }^{3} J_{\mathrm{PH}}=7.0,2 \mathrm{H},\left(\mathrm{CH}_{3} \underline{\mathrm{H}}_{2} \mathrm{O}\right)_{\mathrm{A}}\right), 4.90(\mathrm{~d}$, $\left.{ }^{2} J_{\mathrm{PH}}=13.9,1 \mathrm{H}, \mathrm{CHCl}\right), 7.19\left(\mathrm{~d},{ }^{3} J_{\mathrm{HH}}=7.9,2 \mathrm{H}, \underline{\mathrm{H}}_{\text {meta }}\right.$ of $\left.\mathrm{C}_{6} \mathrm{H}_{4}\right), 7.44\left(\mathrm{dd},{ }^{3} J_{\mathrm{HH}}=7.9,{ }^{4} J_{\mathrm{PH}}=1.6,2 \mathrm{H}, \underline{\mathrm{H}}_{\mathrm{ortho}}\right.$ of $\left.\mathrm{C}_{6} \mathrm{H}_{4}\right) . /{ }^{13} \mathrm{C}-\mathrm{NMR} 16.1\left(\mathrm{~d},{ }^{3} J_{\mathrm{PC}}=6.5,\left(\underline{\mathrm{CH}}_{3} \mathrm{CH}_{2} \mathrm{O}\right)_{\mathrm{A}}\right), 16.3\left(\mathrm{~d},{ }^{3} \mathrm{~J}_{\mathrm{PC}}=6.1,\left(\underline{\mathrm{CH}}_{3} \mathrm{CH}_{2} \mathrm{O}\right)_{\mathrm{B}}\right), 21.1\left(\mathrm{~s}, \mathrm{C}_{6} \mathrm{H}_{4} \underline{\mathrm{CH}}_{3}\right)$, $53.3\left(\mathrm{~d},{ }^{1} J_{\mathrm{PC}}=161.2, \underline{\mathrm{CHCl}}\right), 63.7\left(\mathrm{t},{ }^{2} J_{\mathrm{PC}}=6.7,\left(\mathrm{CH}_{3} \underline{\mathrm{CH}}_{2} \mathrm{O}\right)_{\mathrm{A}}\right), 63.9\left(\mathrm{t},{ }^{2} J_{\mathrm{PC}}=6.7,\left(\mathrm{CH}_{3} \underline{C H}_{2} \mathrm{O}\right)_{\mathrm{B}}\right), 128.8(\mathrm{~d}$, ${ }^{3} J_{\mathrm{PC}}=6.1, \underline{\mathrm{C}}_{\text {ortho }}$ of $\left.\mathrm{C}_{6} \mathrm{H}_{4}\right), 129.1\left(\mathrm{~s}, \underline{\mathrm{C}}_{\text {meta }}\right.$ of $\left.\mathrm{C}_{6} \mathrm{H}_{4}\right), 131.1\left(\mathrm{~d},{ }^{2} J_{\mathrm{PC}}=3.2, \underline{\mathrm{C}}_{\text {ipso }}\right.$ of $\left.\mathrm{C}_{6} \mathrm{H}_{4}\right), 138.8\left(\mathrm{~s}, \underline{\mathrm{C}}_{\text {para }}\right.$ of $\left.\mathrm{C}_{6} \mathrm{H}_{4}\right) . / \mathbf{~ m} / \mathbf{z}(\mathrm{CI}): 277\left(\mathrm{M}+1{ }^{35} \mathrm{Cl}, 19\right), 279\left(\mathrm{M}+1{ }^{37} \mathrm{Cl}, 6\right), 294\left(\mathrm{M}+18{ }^{35} \mathrm{Cl}, 100\right), 296\left(\mathrm{M}+18{ }^{37} \mathrm{Cl}, 38\right)$.

Diethyl $\alpha$-chloro-4-fluorobenzylphosphonate (5e) $\mathrm{C}_{11} \mathrm{H}_{15} \mathrm{O}_{3} \mathrm{FClP}(\mathrm{M}=280.5)$ : 31P-NMR +17.0 (s). / 19F-NMR -112.5 (s, $\left.\mathrm{C}_{6} \mathrm{H}_{4} \underline{\mathrm{F}}\right)$. / 1 $\mathbf{H}-\mathrm{NMR} 1.00\left(\mathrm{t},{ }^{3} \mathrm{~J}_{\mathrm{HH}}=7.0,3 \mathrm{H},\left(\mathrm{C}_{3} \mathrm{CH}_{2} \mathrm{O}\right)_{\mathrm{A}}\right), 1.14\left(\mathrm{t},{ }^{3} J_{\mathrm{HH}}=7.0,3 \mathrm{H}\right.$, $\left.\left(\mathrm{CH}_{3} \mathrm{CH}_{2} \mathrm{O}\right)_{\mathrm{B}}\right), 3.67-3.93\left(\mathrm{~m}, 2 \mathrm{H},\left(\mathrm{CH}_{3} \mathrm{CH}_{2} \mathrm{O}\right)_{\mathrm{B}}\right), 4.02\left(\mathrm{p},{ }^{3} J_{\mathrm{HH}^{3}}{ }^{3} J_{\mathrm{PH}}=7.0,2 \mathrm{H},\left(\mathrm{CH}_{3} \mathrm{CH}_{2} \mathrm{O}\right)_{\mathrm{A}}\right), 4.75(\mathrm{~d}$, $\left.{ }^{2} J_{\mathrm{PH}}=14.1,1 \mathrm{H}, \mathrm{CHCl}\right), 6.87\left(\mathrm{t},{ }^{3} J_{\mathrm{HH}}={ }^{3} J_{\mathrm{FH}}=8.7,2 \mathrm{H}, \underline{\mathrm{H}}_{\text {meta }}\right.$ of $\left.\mathrm{C}_{6} \mathrm{H}_{4}\right), 7.32-7.50\left(\mathrm{~m}, 2 \mathrm{H}, \underline{\mathrm{H}}_{\mathrm{ortho}}\right.$ of $\left.\mathrm{C}_{6} \mathrm{H}_{4}\right)$. / ${ }^{13} \mathrm{C}$-NMR $16.5\left(\mathrm{~d},{ }^{3} J_{\mathrm{PC}}=6.8,\left(\mathrm{CH}_{3} \mathrm{CH}_{2} \mathrm{O}\right)_{\mathrm{A}}\right), 16.7\left(\mathrm{~d},{ }^{3} J_{\mathrm{PC}}=6.1,\left(\mathrm{CH}_{3} \mathrm{CH}_{2} \mathrm{O}\right)_{\mathrm{B}}\right), 53.1\left(\mathrm{~d},{ }^{1} J_{\mathrm{PC}}=160.6, \underline{\mathrm{CHCl}}\right)$, $64.2\left(\mathrm{~d},{ }^{2} J_{\mathrm{PC}}=7.1,\left(\mathrm{CH}_{3} \underline{\mathrm{CH}}{ }_{2} \mathrm{O}\right)_{\mathrm{B}}\right), 64.5\left(\mathrm{~d},{ }^{2} J_{\mathrm{PC}}=6.9,\left(\mathrm{CH}_{3} \underline{\mathrm{CH}_{2} \mathrm{O}}\right)_{\mathrm{A}}\right), 115.9\left(\mathrm{~d},{ }^{2} J_{\mathrm{FC}}=21.3, \underline{\mathrm{C}}_{\text {meta }}\right.$ of $\left.\mathrm{C}_{6} \mathrm{H}_{4}\right)$, $130.5\left(\mathrm{~d},{ }^{2} J_{\mathrm{PC}}=3.0, \underline{\mathrm{C}}_{\mathrm{ipso}}\right.$ of $\left.\mathrm{C}_{6} \mathrm{H}_{4}\right), 131.2\left(\mathrm{t},{ }^{3} J_{\mathrm{PC}}={ }^{3} J_{\mathrm{FC}}=7.5, \underline{\mathrm{C}}_{\text {ortho }}\right.$ of $\left.\mathrm{C}_{6} \mathrm{H}_{4}\right), 163.3\left(\mathrm{dd},{ }^{5} J_{\mathrm{PC}}=2.8\right.$, ${ }^{1} J_{\mathrm{FC}}=248.8, \underline{\mathrm{C}}_{\text {para }}$ of $\left.\mathrm{C}_{6} \mathrm{H}_{4}\right) . / \mathbf{m} / \mathbf{z}(\mathrm{CI}): 281\left(\mathrm{M}+1{ }^{35} \mathrm{Cl}, 100\right), 283\left(\mathrm{M}+1{ }^{37} \mathrm{Cl}, 33\right), 298\left(\mathrm{M}+18{ }^{35} \mathrm{Cl}, 39\right), 300$ $\left(\mathrm{M}+18{ }^{37} \mathrm{Cl}, 11\right)$.

Diethyl $\alpha$-chloro-4-chlorobenzylphosphonate (5f) ${ }^{15} \mathrm{C}_{11} \mathrm{H}_{15} \mathrm{O}_{3} \mathrm{Cl}_{2} \mathrm{P}(\mathrm{M}=297)$ : ${ }^{31} \mathbf{P}-\mathbf{N M R}+16.4$ (s). / 1 $\mathbf{H}-$ NMR $1.10\left(\mathrm{t},{ }^{3} \mathrm{~J}_{\mathrm{HH}}=7.0,3 \mathrm{H},\left(\mathrm{C}_{3} \mathrm{CH}_{2} \mathrm{O}\right)_{\mathrm{A}}\right), 1.22\left(\mathrm{t},{ }^{3} \mathrm{~J}_{\mathrm{HH}}=7.0,3 \mathrm{H},\left(\mathrm{C}_{3} \mathrm{CH}_{2} \mathrm{O}\right)_{\mathrm{B}}\right), 3.78-4.01(\mathrm{~m}, 2 \mathrm{H}$, $\left.\left(\mathrm{CH}_{3} \underline{\mathrm{C}}_{2} \mathrm{O}\right)_{\mathrm{B}}\right), 4.08\left(\mathrm{p},{ }^{3} J_{\mathrm{HH}}={ }^{3} J_{\mathrm{PH}}=7.0,2 \mathrm{H},\left(\mathrm{CH}_{3} \underline{\mathrm{CH}}_{2} \mathrm{O}\right)_{\mathrm{A}}\right), 4.79\left(\mathrm{~d},{ }^{2} J_{\mathrm{PH}}=14.3,1 \mathrm{H}, \mathrm{C} \underline{\mathrm{HCl}}\right), 7.24(\mathrm{~d}$, ${ }^{3} J_{\mathrm{HH}}=8.5,2 \mathrm{H}, \underline{\mathrm{H}}_{\text {meta }}$ of $\left.\mathrm{C}_{6} \mathrm{H}_{4}\right), 7.44\left(\mathrm{dd},{ }^{3} J_{\mathrm{HH}}=8.5,{ }^{4} J_{\mathrm{PH}}=1.6,2 \mathrm{H}, \underline{\mathrm{H}}_{\mathrm{ortho}}\right.$ of $\left.\mathrm{C}_{6} \mathrm{H}_{4}\right) . / 13 \mathrm{C}-\mathrm{NMR} 16.6(\mathrm{~d}$,

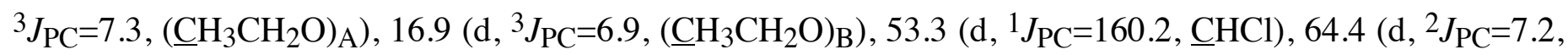
$\left.\left(\mathrm{CH}_{3} \underline{\mathrm{CH}}_{2} \mathrm{O}\right)_{\mathrm{A}}\right), 64.7\left(\mathrm{~d},{ }^{2} J_{\mathrm{PC}}=6.4,\left(\mathrm{CH}_{3} \underline{\mathrm{CH}}_{2} \mathrm{O}\right)_{\mathrm{B}}\right), 129.2\left(\mathrm{~s}, \underline{\mathrm{C}}_{\text {meta }}\right.$ of $\left.\mathrm{C}_{6} \mathrm{H}_{4}\right), 130.7$ (d, ${ }^{3} J_{\mathrm{PC}}=6.0, \underline{\mathrm{C}}_{\text {ortho }}$ of $\left.\mathrm{C}_{6} \mathrm{H}_{4}\right), 133.3\left(\mathrm{~d},{ }^{2} J_{\mathrm{PC}}=3.0, \underline{\mathrm{C}}_{\mathrm{ipso}}\right.$ of $\left.\mathrm{C}_{6} \mathrm{H}_{4}\right), 135.4\left(\mathrm{~d},{ }^{5} J_{\mathrm{PC}}=2.8, \underline{\mathrm{C}}_{\mathrm{para}}\right.$ of $\left.\mathrm{C}_{6} \mathrm{H}_{4}\right) . / \mathbf{m} / \mathbf{z}(\mathrm{CI}): 297(\mathrm{M}+12 * 35 \mathrm{Cl}$, 100), $299\left(\mathrm{M}+1{ }^{35} \mathrm{Cl}+{ }^{37} \mathrm{Cl}, 69\right), 301(\mathrm{M}+12 * 37 \mathrm{Cl}, 14), 314(\mathrm{M}+182 * 35 \mathrm{Cl}, 67), 316\left(\mathrm{M}+18{ }^{35} \mathrm{Cl}+{ }^{37} \mathrm{Cl}, 47\right)$, $318(\mathrm{M}+182 * 37 \mathrm{Cl}, 9)$.

Diethyl $\alpha$-chloro-4-bromobenzylphosphonate $(\mathbf{5 g})^{15} \mathrm{C}_{11} \mathrm{H}_{15} \mathrm{O}_{3} \mathrm{ClBrP}(\mathrm{M}=341.5):{ }^{31} \mathbf{P}-\mathbf{N M R}+16.4$ (s). / 1H-NMR $1.03\left(\mathrm{t},{ }^{3} \mathrm{~J}_{\mathrm{HH}}=7.1,3 \mathrm{H},\left(\mathrm{CH}_{3} \mathrm{CH}_{2} \mathrm{O}\right)_{\mathrm{A}}\right), 1.15\left(\mathrm{t},{ }^{3} \mathrm{~J}_{\mathrm{HH}}=7.1,3 \mathrm{H},\left(\mathrm{CH}_{3} \mathrm{CH}_{2} \mathrm{O}\right)_{\mathrm{B}}\right), 3.71-3.94(\mathrm{~m}, 2 \mathrm{H}$, $\left.\left(\mathrm{CH}_{3} \underline{\mathrm{C}}_{2} \mathrm{O}\right)_{\mathrm{B}}\right), 4.03\left(\mathrm{p},{ }^{3} J_{\mathrm{HH}}={ }^{3} J_{\mathrm{PH}}=7.1,2 \mathrm{H},\left(\mathrm{CH}_{3} \underline{\mathrm{C}}_{2} \mathrm{O}\right)_{\mathrm{A}}\right), 4.75\left(\mathrm{~d},{ }^{2} J_{\mathrm{PH}}=14.3,1 \mathrm{H}, \mathrm{C} \underline{\mathrm{HCl}}\right), 7.25(\mathrm{dd}$, ${ }^{3} J_{\mathrm{HH}}=8.7,{ }^{4} J_{\mathrm{PH}}=1.7,2 \mathrm{H}, \underline{\mathrm{H}}_{\text {ortho }}$ of $\left.\mathrm{C}_{6} \mathrm{H}_{4}\right), 7.33\left(\mathrm{~d},{ }^{3} J_{\mathrm{HH}}=8.7,2 \mathrm{H}, \underline{\mathrm{H}}_{\text {meta }}\right.$ of $\left.\mathrm{C}_{6} \mathrm{H}_{4}\right) . / 13 \mathbf{C}-\mathbf{N M R} 16.5(\mathrm{~d}$, 
$\left.{ }^{3} J_{\mathrm{PC}}=7.5,\left(\underline{\mathrm{CH}}_{3} \mathrm{CH}_{2} \mathrm{O}\right)_{\mathrm{A}}\right), 16.7\left(\mathrm{~d},{ }^{3} J_{\mathrm{PC}}=6.7,\left(\underline{\mathrm{CH}}_{3} \mathrm{CH}_{2} \mathrm{O}\right)_{\mathrm{B}}\right), 53.1\left(\mathrm{~d},{ }^{1} J_{\mathrm{PC}}=159.5, \underline{\mathrm{CHCl}}\right), 64.2\left(\mathrm{~d},{ }^{2} J_{\mathrm{PC}}=6.7\right.$, $\left.\left(\mathrm{CH}_{3} \underline{\mathrm{CH}}_{2} \mathrm{O}\right)_{\mathrm{A}}\right), 64.5\left(\mathrm{~d},{ }^{2} J_{\mathrm{PC}}=6.5,\left(\mathrm{CH}_{3} \underline{\mathrm{CH}}_{2} \mathrm{O}\right)_{\mathrm{B}}\right), 123.3\left(\mathrm{~d},{ }^{5} J_{\mathrm{PC}}=3.0, \underline{\mathrm{C}}_{\mathrm{para}}\right.$ of $\left.\mathrm{C}_{6} \mathrm{H}_{4}\right), 130.8\left(\mathrm{~d},{ }^{3} J_{\mathrm{PC}}=6.0\right.$,

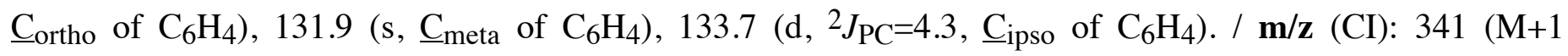
$\left.{ }^{35} \mathrm{Cl}+{ }^{79} \mathrm{Br}, 73\right), 343\left(\mathrm{M}+1{ }^{37} \mathrm{Cl}+{ }^{79} \mathrm{Br} ;{ }^{35} \mathrm{Cl}+{ }^{81} \mathrm{Br}, 100\right), 345\left(\mathrm{M}+1{ }^{37} \mathrm{Cl}+{ }^{81} \mathrm{Br}, 23\right), 358\left(\mathrm{M}+18{ }^{35} \mathrm{Cl}+{ }^{79} \mathrm{Br}\right.$, 53), $360\left(\mathrm{M}+18{ }^{37} \mathrm{Cl}+{ }^{79} \mathrm{Br} ;{ }^{35} \mathrm{Cl}+{ }^{81} \mathrm{Br}, 69\right), 362\left(\mathrm{M}+18{ }^{37} \mathrm{Cl}+{ }^{81} \mathrm{Br}, 17\right)$.

Diethyl $\alpha$-chloro-4-methoxybenzylphosphonate (5h) $\mathrm{C}_{12} \mathrm{H}_{18} \mathrm{O}_{4} \mathrm{ClP}(\mathrm{M}=292.5)$ : 31P-NMR +17.6 (s). / 1H-NMR $1.23\left(\mathrm{t},{ }^{3} J_{\mathrm{HH}}=7.1,3 \mathrm{H},\left(\mathrm{C}_{3} \mathrm{CH}_{2} \mathrm{O}\right)_{\mathrm{A}}\right), 1.38\left(\mathrm{t},{ }^{3} J_{\mathrm{HH}}=7.0,3 \mathrm{H},\left(\mathrm{C}_{3} \mathrm{CH}_{2} \mathrm{O}\right)_{\mathrm{B}}\right), 3.85\left(\mathrm{~s}, 3 \mathrm{H}, \mathrm{OC}_{3}\right)$, 3.87-4.16 (m, 2H, $\left.\left(\mathrm{CH}_{3} \mathrm{CH}_{2} \mathrm{O}\right)_{\mathrm{B}}\right), 4.24\left(\mathrm{p},{ }^{3} J_{\mathrm{HH}}={ }^{3} J_{\mathrm{PH}}=7.1,2 \mathrm{H},\left(\mathrm{CH}_{3} \mathrm{CH}_{2} \mathrm{O}\right)_{\mathrm{A}}\right), 4.93\left(\mathrm{~d},{ }^{2} J_{\mathrm{PH}}=13.7,1 \mathrm{H}\right.$, $\mathrm{C} \underline{\mathrm{HCl}}), 6.93\left(\mathrm{~d},{ }^{3} J_{\mathrm{HH}}=8.2,2 \mathrm{H}, \underline{\mathrm{H}}_{\text {meta }}\right.$ of $\left.\mathrm{C}_{6} \mathrm{H}_{4}\right), 7.51\left(\mathrm{dd},{ }^{3} J_{\mathrm{HH}}=8.8,{ }^{4} J_{\mathrm{PH}}=2.0,2 \mathrm{H}, \underline{\mathrm{H}}_{\text {ortho }}\right.$ of $\left.\mathrm{C}_{6} \mathrm{H}_{4}\right)$. / ${ }^{13} \mathrm{C}$-NMR $16.8\left(\mathrm{~d},{ }^{3} J_{\mathrm{PC}}=6.4,\left(\mathrm{CH}_{3} \mathrm{CH}_{2} \mathrm{O}\right)_{\mathrm{A}}\right), 17.0\left(\mathrm{~d},{ }^{3} J_{\mathrm{PC}}=4.6,\left(\mathrm{CH}_{3} \mathrm{CH}_{2} \mathrm{O}\right)_{\mathrm{B}}\right), 53.9\left(\mathrm{~d},{ }^{1} J_{\mathrm{PC}}=162.0, \underline{\mathrm{CHCl}}\right)$, $55.8\left(\mathrm{~s}, \mathrm{O}_{\mathrm{CH}_{3}}\right), 64.4\left(\mathrm{t},{ }^{2} J_{\mathrm{PC}}=7.2,\left(\mathrm{CH}_{3} \underline{\mathrm{CH}}_{2} \mathrm{O}\right)_{\mathrm{A}}\right), 64.6\left(\mathrm{t},{ }^{2} J_{\mathrm{PC}}=7.2,\left(\mathrm{CH}_{3} \underline{\mathrm{CH}}_{2} \mathrm{O}\right)_{\mathrm{B}}\right), 114.6\left(\mathrm{~s}, \underline{\mathrm{C}}_{\text {meta }}\right.$ of $\left.\mathrm{C}_{6} \mathrm{H}_{4}\right), 126.7\left(\mathrm{~d},{ }^{2} J_{\mathrm{PC}}=4.0, \underline{\mathrm{C}}_{\mathrm{ipso}}\right.$ of $\left.\mathrm{C}_{6} \mathrm{H}_{4}\right), 130.8\left(\mathrm{~d},{ }^{3} J_{\mathrm{PC}}=6.2, \underline{\mathrm{C}}_{\text {ortho }}\right.$ of $\left.\mathrm{C}_{6} \mathrm{H}_{4}\right), 160.7\left(\mathrm{~d},{ }^{5} J_{\mathrm{PC}}=2.6, \underline{\mathrm{C}}_{\text {para }}\right.$ of $\left.\mathrm{C}_{6} \mathrm{H}_{4}\right) . / \mathbf{~ m} / \mathbf{z}(\mathrm{CI}): 293\left(\mathrm{M}+1{ }^{35} \mathrm{Cl}, 100\right), 295\left(\mathrm{M}+1{ }^{37} \mathrm{Cl}, 32\right), 310\left(\mathrm{M}+18{ }^{35} \mathrm{Cl}, 15\right), 312\left(\mathrm{M}+18{ }^{37} \mathrm{Cl}, 6\right)$.

Diethyl $\alpha$-bromobenzylphosphonate $(\mathbf{6 a})^{10,16,30} \mathrm{C}_{11} \mathrm{H}_{16} \mathrm{O}_{3} \mathrm{BrP}(\mathrm{M}=307)$ : 31P-NMR +17.3 (s). / 1H-NMR $1.07\left(\mathrm{t},{ }^{3} J_{\mathrm{HH}}=7.0,3 \mathrm{H},\left(\mathrm{CH}_{3} \mathrm{CH}_{2} \mathrm{O}\right)_{\mathrm{A}}\right), 1.26\left(\mathrm{t},{ }^{3} J_{\mathrm{HH}}=7.0,3 \mathrm{H},\left(\mathrm{CH}_{3} \mathrm{CH}_{2} \mathrm{O}\right)_{\mathrm{B}}\right), 3.73-4.04(\mathrm{~m}, 2 \mathrm{H}$, $\left.\left(\mathrm{CH}_{3} \underline{\mathrm{H}}_{2} \mathrm{O}\right)_{\mathrm{B}}\right), 4.16\left(\mathrm{p},{ }^{3} J_{\mathrm{HH}}={ }^{3} J_{\mathrm{PH}}=7.0,2 \mathrm{H},\left(\mathrm{CH}_{3} \underline{\mathrm{H}}_{2} \mathrm{O}\right)_{\mathrm{A}}\right), 4.81\left(\mathrm{~d},{ }^{2} J_{\mathrm{PH}}=13.0,1 \mathrm{H}, \mathrm{CHBr}\right), 7.22-7.30(\mathrm{~m}$, $3 \mathrm{H}, \underline{\mathrm{H}}_{\text {meta,para }}$ of $\left.\mathrm{C}_{6} \mathrm{H}_{5}\right), 7.46-7.52\left(\mathrm{~m}, 2 \mathrm{H}, \underline{\mathrm{H}}_{\text {ortho }}\right.$ of $\left.\mathrm{C}_{6} \mathrm{H}_{5}\right)$. / ${ }^{13} \mathrm{C}-\mathrm{NMR} 16.7\left(\mathrm{~d},{ }^{3} \mathrm{~J}_{\mathrm{PC}}=5.1,\left(\mathrm{CH}_{3} \mathrm{CH}_{2} \mathrm{O}\right)_{\mathrm{A}}\right)$,

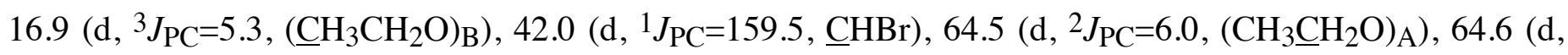
$\left.{ }^{2} J_{\mathrm{PC}}=6.3,\left(\mathrm{CH}_{3} \underline{C H}_{2} \mathrm{O}\right)_{\mathrm{B}}\right), 129.2\left(\mathrm{~s}, \underline{\mathrm{C}}_{\text {meta }}\right.$ of $\left.\mathrm{C}_{6} \mathrm{H}_{5}\right), 129.7\left(\mathrm{~s}, \underline{\mathrm{C}}_{\text {para }}\right.$ of $\left.\mathrm{C}_{6} \mathrm{H}_{5}\right), 130.0\left(\mathrm{~d},{ }^{3} J_{\mathrm{PC}}=7.1, \underline{\mathrm{C}}_{\mathrm{ortho}}\right.$ of $\left.\mathrm{C}_{6} \mathrm{H}_{5}\right), 135.1\left(\mathrm{~d},{ }^{2} J_{\mathrm{PC}}=3.0, \underline{\mathrm{C}}_{\mathrm{ipso}}\right.$ of $\left.\mathrm{C}_{6} \mathrm{H}_{5}\right) . / \mathbf{m} / \mathbf{z}(\mathrm{CI}): 307\left(\mathrm{M}+1{ }^{79} \mathrm{Br}, 100\right), 309\left(\mathrm{M}+1{ }^{81} \mathrm{Br}, 100\right), 324$ $\left(\mathrm{M}+18^{79} \mathrm{Br}, 55\right), 326\left(\mathrm{M}+18^{81} \mathrm{Br}, 55\right)$.

Diethyl $\alpha$-bromo-2-fluorobenzylphosphonate (6b) $\mathrm{C}_{11} \mathrm{H}_{15} \mathrm{O}_{3} \mathrm{FBrP} \quad(\mathrm{M}=325)$ : 31P-NMR +16.2 (s). /

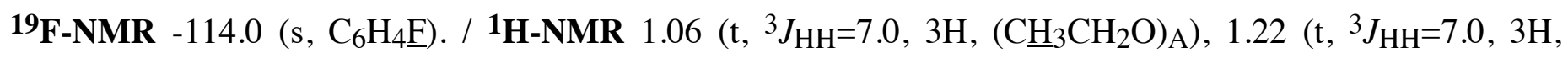
$\left.\left(\mathrm{CH}_{3} \mathrm{CH}_{2} \mathrm{O}\right)_{\mathrm{B}}\right), 3.99\left(\mathrm{p},{ }^{3} J_{\mathrm{HH}}={ }^{3} J_{\mathrm{PH}}=7.0,2 \mathrm{H},\left(\mathrm{CH}_{3} \mathrm{CH}_{2} \mathrm{O}\right)_{\mathrm{B}}\right), 4.14\left(\mathrm{p},{ }^{3} J_{\mathrm{HH}^{3}}{ }^{3} J_{\mathrm{PH}}=7.0,2 \mathrm{H},\left(\mathrm{CH}_{3} \mathrm{CH}_{2} \mathrm{O}\right)_{\mathrm{A}}\right)$,

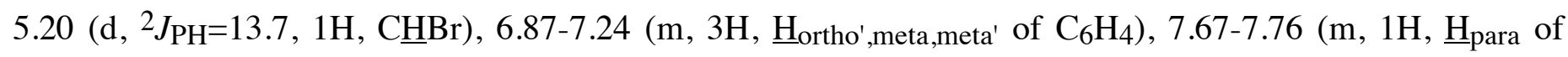

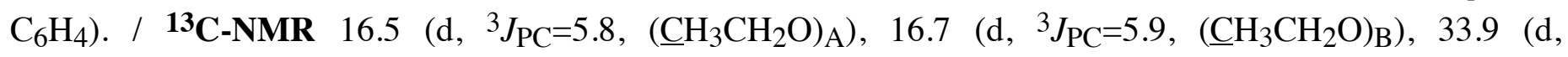
$\left.{ }^{1} J_{\mathrm{PC}}=164.5, \underline{\mathrm{CHBr}}\right), 64.3\left(\mathrm{~d},{ }^{2} J_{\mathrm{PC}}=7.8,\left(\mathrm{CH}_{3} \underline{\mathrm{C}} \mathrm{H}_{2} \mathrm{O}\right)_{\mathrm{B}}\right), 64.6\left(\mathrm{~d},{ }^{2} J_{\mathrm{PC}}=5.3,\left(\mathrm{CH}_{3} \underline{\mathrm{CH}}{ }_{2} \mathrm{O}\right)_{\mathrm{A}}\right), 115.7(\mathrm{~d}$, ${ }^{2} J_{\mathrm{FC}}=22.3, \underline{\mathrm{C}}_{\text {meta }}$ of $\left.\mathrm{C}_{6} \mathrm{H}_{4}\right), 122.5\left(\mathrm{~d},{ }^{2} J_{\mathrm{PC}}=14.7, \underline{\mathrm{C}}_{\mathrm{ipso}}\right.$ of $\left.\mathrm{C}_{6} \mathrm{H}_{4}\right), 125.2\left(\mathrm{~d},{ }^{4} J_{\mathrm{FC}}=2.9, \underline{\mathrm{C}}_{\text {meta' }}\right.$ of $\left.\mathrm{C}_{6} \mathrm{H}_{4}\right), 131.2$ $\left(\mathrm{d},{ }^{3} J_{\mathrm{FC}}=2.5, \underline{\mathrm{C}}_{\text {ortho' }}\right.$ of $\left.\mathrm{C}_{6} \mathrm{H}_{4}\right), 132.2\left(\mathrm{~s}, \underline{\mathrm{C}}_{\text {para }}\right.$ of $\left.\mathrm{C}_{6} \mathrm{H}_{4}\right), 159.9\left(\mathrm{dd},{ }^{3} J_{\mathrm{PC}}=9.1,{ }^{1} J_{\mathrm{FC}}=248.8, \underline{\mathrm{C}}_{\text {ortho }}\right.$ of $\left.\mathrm{C}_{6} \mathrm{H}_{4}\right) . /$ $\mathbf{m} / \mathbf{z}(\mathrm{CI}): 325\left(\mathrm{M}+1{ }^{79} \mathrm{Br}, 100\right), 327\left(\mathrm{M}+1{ }^{81} \mathrm{Br}, 100\right), 342\left(\mathrm{M}+18^{79} \mathrm{Br}, 77\right), 344\left(\mathrm{M}+18{ }^{81} \mathrm{Br}, 10\right)$.

Diethyl $\alpha$-bromo-4-methylbenzylphosphonate (6c) $\mathrm{C}_{12} \mathrm{H}_{18} \mathrm{O}_{3} \mathrm{BrP}(\mathrm{M}=321)$ : 31P-NMR +17.6 (s). / 1H-NMR $1.16\left(\mathrm{t},{ }^{3} J_{\mathrm{HH}}=7.1,3 \mathrm{H},\left(\mathrm{CH}_{3} \mathrm{CH}_{2} \mathrm{O}\right)_{\mathrm{A}}\right), 1.33\left(\mathrm{t},{ }^{3} \mathrm{~J}_{\mathrm{HH}}=7.1,3 \mathrm{H},\left(\mathrm{CH}_{3} \mathrm{CH}_{2} \mathrm{O}\right)_{\mathrm{B}}\right), 2.33\left(\mathrm{~s}, 3 \mathrm{H}, \mathrm{C}_{6} \mathrm{H}_{4} \mathrm{CH}_{3}\right), 3.72-$ $4.12\left(\mathrm{~m}, 2 \mathrm{H},\left(\mathrm{CH}_{3} \underline{\mathrm{H}}_{2} \mathrm{O}\right)_{\mathrm{B}}\right), 4.21\left(\mathrm{p},{ }^{3} J_{\mathrm{HH}}={ }^{3} J_{\mathrm{PH}}=7.1,2 \mathrm{H},\left(\mathrm{CH}_{3} \underline{\mathrm{C}}_{2} \mathrm{O}\right)_{\mathrm{A}}\right), 4.87\left(\mathrm{~d},{ }^{2} J_{\mathrm{PH}}=12.8,1 \mathrm{H}, \mathrm{C} \underline{\mathrm{HBr}}\right)$, $7.15\left(\mathrm{~d},{ }^{3} \mathrm{~J}_{\mathrm{HH}}=8.0,2 \mathrm{H}, \underline{\mathrm{H}}_{\text {meta }}\right.$ of $\left.\mathrm{C}_{6} \mathrm{H}_{4}\right), 7.45\left(\mathrm{dd},{ }^{3} \mathrm{~J}_{\mathrm{HH}}=8.0,{ }^{4} \mathrm{~J}_{\mathrm{PH}}=1.6,2 \mathrm{H}, \underline{\mathrm{H}}_{\text {ortho }}\right.$ of $\left.\mathrm{C}_{6} \mathrm{H}_{4}\right) . / 13 \mathrm{C}-\mathrm{NMR} 16.7$ $\left(\mathrm{d},{ }^{3} J_{\mathrm{PC}}=5.9,\left(\underline{\mathrm{CH}}_{3} \mathrm{CH}_{2} \mathrm{O}\right)_{\mathrm{A}}\right), 16.9\left(\mathrm{~d},{ }^{3} J_{\mathrm{PC}}=6.0,\left(\underline{\mathrm{CH}}_{3} \mathrm{CH}_{2} \mathrm{O}\right)_{\mathrm{B}}\right), 21.8\left(\mathrm{~s}, \mathrm{C}_{6} \mathrm{H}_{4} \underline{\mathrm{CH}}_{3}\right), 41.9\left(\mathrm{~d},{ }^{1} J_{\mathrm{PC}}=161.2\right.$, $\underline{\mathrm{CHBr}}$ ), 64.7 (d, $\left.{ }^{2} \mathrm{~J}_{\mathrm{PC}}=6.0, \mathrm{CH}_{3} \underline{\mathrm{CH}}_{2} \mathrm{O}\right), 129.8$ (s, $\underline{\mathrm{C}}_{\text {ortho }}$ of $\left.\mathrm{C}_{6} \mathrm{H}_{4}\right), 129.9$ (s, $\underline{\mathrm{C}}_{\text {meta }}$ of $\left.\mathrm{C}_{6} \mathrm{H}_{4}\right), 131.9$ (d, ${ }^{2} J_{\mathrm{PC}}=3.8, \underline{\mathrm{C}}_{\mathrm{ipso}}$ of $\left.\mathrm{C}_{6} \mathrm{H}_{4}\right), 139.6\left(\mathrm{~d},{ }^{5} J_{\mathrm{PC}}=2.8, \underline{\mathrm{C}}_{\mathrm{para}}\right.$ of $\left.\mathrm{C}_{6} \mathrm{H}_{4}\right) . / \mathbf{m} / \mathbf{z}(\mathrm{CI}): 321\left(\mathrm{M}+1{ }^{79} \mathrm{Br}, 100\right), 323(\mathrm{M}+1$ $\left.{ }^{81} \mathrm{Br}, 100\right), 338\left(\mathrm{M}+18{ }^{79} \mathrm{Br}, 30\right), 340\left(\mathrm{M}+18^{81} \mathrm{Br}, 30\right)$. 
Diethyl $\alpha$-bromo-4-fluorobenzylphosphonate (6d) $\mathrm{C}_{11} \mathrm{H}_{15} \mathrm{O}_{3} \mathrm{FBrP} \quad(\mathrm{M}=325)$ : 31P-NMR +17.4 (s). / ${ }^{19}$ F-NMR -115.5 (s, $\left.\mathrm{C}_{6} \mathrm{H}_{4} \mathrm{~F}\right)$. / 1 $\mathbf{H}-\mathbf{N M R} 1.00\left(\mathrm{t},{ }^{3} \mathrm{~J}_{\mathrm{HH}}=7.0,3 \mathrm{H},\left(\mathrm{CH}_{3} \mathrm{CH}_{2} \mathrm{O}\right)_{\mathrm{A}}\right), 1.18\left(\mathrm{t},{ }^{3} J_{\mathrm{HH}}=7.0,3 \mathrm{H}\right.$, $\left.\left(\mathrm{C}_{3} \mathrm{CH}_{2} \mathrm{O}\right)_{\mathrm{B}}\right), 3.64-3.99\left(\mathrm{~m}, 2 \mathrm{H},\left(\mathrm{CH}_{3} \underline{\mathrm{C}}_{2} \mathrm{O}\right)_{\mathrm{B}}\right), 4.02\left(\mathrm{p},{ }^{3} \mathrm{~J}_{\mathrm{HH}}={ }^{3} J_{\mathrm{PH}}=7.0,2 \mathrm{H},\left(\mathrm{CH}_{3} \mathrm{C} \underline{H}_{2} \mathrm{O}\right)_{\mathrm{A}}\right), 4.74(\mathrm{~d}$, $\left.{ }^{2} J_{\mathrm{PH}}=13.1,1 \mathrm{H}, \mathrm{CHBr}\right), 6.88\left(\mathrm{t},{ }^{3} J_{\mathrm{HH}}={ }^{3} J_{\mathrm{FH}}=8.6,2 \mathrm{H}, \underline{\mathrm{H}}_{\text {meta }}\right.$ of $\left.\mathrm{C}_{6} \mathrm{H}_{4}\right), 7.38-7.46\left(\mathrm{~m}, 2 \mathrm{H}, \underline{\mathrm{H}}_{\mathrm{ortho}}\right.$ of $\left.\mathrm{C}_{6} \mathrm{H}_{4}\right)$. / ${ }^{13} \mathrm{C}$-NMR $16.5\left(\mathrm{~d},{ }^{3} J_{\mathrm{PC}}=6.0,\left(\underline{\mathrm{CH}}_{3} \mathrm{CH}_{2} \mathrm{O}\right)_{\mathrm{A}}\right), 16.7\left(\mathrm{~d},{ }^{3} J_{\mathrm{PC}}=6.0,\left(\underline{\mathrm{CH}}_{3} \mathrm{CH}_{2} \mathrm{O}\right)_{\mathrm{B}}\right), 40.9\left(\mathrm{~d},{ }^{1} J_{\mathrm{PC}}=160.3, \underline{\mathrm{CHBr}}\right)$, $64.3\left(\mathrm{~d},{ }^{2} J_{\mathrm{PC}}=6.4,\left(\mathrm{CH}_{3} \mathrm{CH}_{2} \mathrm{O}\right)_{\mathrm{B}}\right), 64.5\left(\mathrm{~d},{ }^{2} J_{\mathrm{PC}}=6.7,\left(\mathrm{CH}_{3} \mathrm{CH}_{2} \mathrm{O}\right)_{\mathrm{A}}\right), 116.0\left(\mathrm{~d},{ }^{2} J_{\mathrm{FC}}=22.1, \underline{\mathrm{C}}_{\text {meta }}\right.$ of $\left.\mathrm{C}_{6} \mathrm{H}_{4}\right)$, $131.1\left(\mathrm{~d},{ }^{2} J_{\mathrm{PC}}=3.0, \underline{\mathrm{C}}_{\mathrm{ipso}}\right.$ of $\left.\mathrm{C}_{6} \mathrm{H}_{4}\right), 131.8\left(\mathrm{t},{ }^{3} J_{\mathrm{PC}}={ }^{3} J_{\mathrm{FC}}=7.6, \underline{\mathrm{C}}_{\text {ortho }}\right.$ of $\left.\mathrm{C}_{6} \mathrm{H}_{4}\right), 163.2\left(\mathrm{dd},{ }^{5} J_{\mathrm{PC}}=3.0\right.$, ${ }^{1} J_{\mathrm{FC}}=251.5, \underline{\mathrm{C}}_{\text {para }}$ of $\left.\mathrm{C}_{6} \mathrm{H}_{4}\right) . / \mathbf{m} / \mathbf{z}(\mathrm{CI}): 325\left(\mathrm{M}+1{ }^{79} \mathrm{Br}, 10\right), 327\left(\mathrm{M}+1{ }^{81} \mathrm{Br}, 10\right), 342\left(\mathrm{M}+18{ }^{79} \mathrm{Br}, 100\right), 344$ $\left(\mathrm{M}+18^{81} \mathrm{Br}, 100\right)$.

Diethyl $\alpha$-bromo-4-chlorobenzylphosphonate (6e) $\mathrm{C}_{11} \mathrm{H}_{15} \mathrm{O}_{3} \mathrm{ClBrP}(\mathrm{M}=341.5)$ : 31P-NMR +16.8 (s). / 1H-NMR $1.07\left(\mathrm{t},{ }^{3} J_{\mathrm{HH}}=7.0,3 \mathrm{H},\left(\mathrm{CH}_{3} \mathrm{CH}_{2} \mathrm{O}\right)_{\mathrm{A}}\right), 1.23\left(\mathrm{t},{ }^{3} \mathrm{~J}_{\mathrm{HH}}=7.0,3 \mathrm{H},\left(\mathrm{CH}_{3} \mathrm{CH}_{2} \mathrm{O}\right)_{\mathrm{B}}\right), 3.75-4.03(\mathrm{~m}, 2 \mathrm{H}$, $\left.\left(\mathrm{CH}_{3} \underline{\mathrm{H}}_{2} \mathrm{O}\right)_{\mathrm{B}}\right), 4.12\left(\mathrm{p},{ }^{3} J_{\mathrm{HH}}={ }^{3} J_{\mathrm{PH}}=7.0,2 \mathrm{H},\left(\mathrm{CH}_{3} \underline{\mathrm{CH}}_{2} \mathrm{O}\right)_{\mathrm{A}}\right), 4.76\left(\mathrm{~d},{ }^{2} J_{\mathrm{PH}}=13.2,1 \mathrm{H}, \mathrm{C} \underline{\mathrm{HBr}}\right), 7.21(\mathrm{~d}$, ${ }^{3} J_{\mathrm{HH}}=8.5,2 \mathrm{H}, \underline{\mathrm{H}}_{\text {meta }}$ of $\left.\mathrm{C}_{6} \mathrm{H}_{4}\right), 7.41\left(\mathrm{dd},{ }^{3} J_{\mathrm{HH}}=8.5,{ }^{4} J_{\mathrm{PH}}=1.6,2 \mathrm{H}, \underline{\mathrm{H}}_{\mathrm{ortho}}\right.$ of $\left.\mathrm{C}_{6} \mathrm{H}_{4}\right)$. / 13C-NMR $16.4(\mathrm{~d}$,

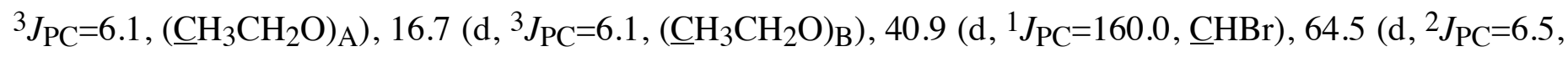
$\left.\left(\mathrm{CH}_{3} \mathrm{CH}_{2} \mathrm{O}\right)_{\mathrm{B}}\right), 64.8\left(\mathrm{~d},{ }^{2} J_{\mathrm{PC}}=7.3,\left(\mathrm{CH}_{3} \underline{\mathrm{CH}}_{2} \mathrm{O}\right)_{\mathrm{A}}\right), 129.3\left(\mathrm{~s}, \underline{\mathrm{C}}_{\text {meta }}\right.$ of $\left.\mathrm{C}_{6} \mathrm{H}_{4}\right), 131.3\left(\mathrm{~d},{ }^{3} J_{\mathrm{PC}}=6.2, \underline{\mathrm{C}}_{\text {ortho }}\right.$ of $\left.\mathrm{C}_{6} \mathrm{H}_{4}\right), 133.7\left(\mathrm{~d},{ }^{2} J_{\mathrm{PC}}=2.8, \underline{\mathrm{C}}_{\text {ipso }}\right.$ of $\left.\mathrm{C}_{6} \mathrm{H}_{4}\right), 135.3\left(\mathrm{~s}, \underline{\mathrm{C}}_{\text {para }}\right.$ of $\left.\mathrm{C}_{6} \mathrm{H}_{4}\right) . / \mathbf{m} / \mathbf{z}(\mathrm{CI}): 341\left(\mathrm{M}+1{ }^{35} \mathrm{Cl}+{ }^{79} \mathrm{Br}, 78\right)$, $343\left(\mathrm{M}+1{ }^{37} \mathrm{Cl}+{ }^{79} \mathrm{Br} ;{ }^{35} \mathrm{Cl}+{ }^{81} \mathrm{Br}, 100\right), 345\left(\mathrm{M}+1{ }^{37} \mathrm{Cl}+{ }^{81} \mathrm{Br}, 25\right), 358\left(\mathrm{M}+18{ }^{35} \mathrm{Cl}+{ }^{79} \mathrm{Br}, 60\right), 360(\mathrm{M}+18$ $\left.{ }^{37} \mathrm{Cl}+{ }^{79} \mathrm{Br} ;{ }^{35} \mathrm{Cl}+{ }^{81} \mathrm{Br}, 78\right), 362\left(\mathrm{M}+18{ }^{37} \mathrm{Cl}+{ }^{81} \mathrm{Br}, 19\right)$.

Diethyl $\alpha$-bromo-4-bromobenzylphosphonate (6f) $\mathrm{C}_{11} \mathrm{H}_{15} \mathrm{O}_{3} \mathrm{Br}_{2} \mathrm{P}(\mathrm{M}=386)$ : 31P-NMR +16.6 (s). / 1 H-NMR $1.03\left(\mathrm{t},{ }^{3} J_{\mathrm{HH}}=7.0,3 \mathrm{H},\left(\mathrm{CH}_{3} \mathrm{CH}_{2} \mathrm{O}\right)_{\mathrm{A}}\right), 1.19\left(\mathrm{t},{ }^{3} \mathrm{~J}_{\mathrm{HH}}=7.0,3 \mathrm{H},\left(\mathrm{CH}_{3} \mathrm{CH}_{2} \mathrm{O}\right)_{\mathrm{B}}\right), 3.71-3.98(\mathrm{~m}, 2 \mathrm{H}$, $\left.\left(\mathrm{CH}_{3} \underline{\mathrm{C}}_{2} \mathrm{O}\right)_{\mathrm{B}}\right), 4.08\left(\mathrm{p},{ }^{3} J_{\mathrm{HH}}={ }^{3} J_{\mathrm{PH}}=7.0,2 \mathrm{H},\left(\mathrm{CH}_{3} \underline{\mathrm{H}}_{2} \mathrm{O}\right)_{\mathrm{A}}\right), 4.70\left(\mathrm{~d},{ }^{2} J_{\mathrm{PH}}=13.2,1 \mathrm{H}, \mathrm{C} \underline{\mathrm{HBr}}\right), 7.23-7.39(\mathrm{~m}$,

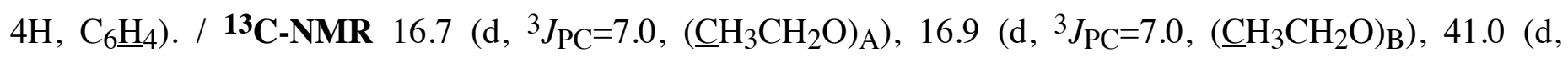
$\left.{ }^{1} J_{\mathrm{PC}}=159.1, \underline{\mathrm{CHBr}}\right), 64.5\left(\mathrm{~d},{ }^{2} J_{\mathrm{PC}}=6.9,\left(\mathrm{CH}_{3} \underline{\mathrm{CH}_{2} \mathrm{O}}\right)_{\mathrm{B}}\right), 64.7\left(\mathrm{~d},{ }^{2} J_{\mathrm{PC}}=7.2,\left(\mathrm{CH}_{3} \underline{\mathrm{CH}_{2} \mathrm{O}}\right)_{\mathrm{A}}\right), 123.4\left(\mathrm{~d},{ }^{5} J_{\mathrm{PC}}=3.0\right.$, $\underline{\mathrm{C}}_{\text {para }}$ of $\left.\mathrm{C}_{6} \mathrm{H}_{4}\right), 131.4\left(\mathrm{~d},{ }^{3} J_{\mathrm{PC}}=6.9, \underline{\mathrm{C}}_{\text {ortho }}\right.$ of $\left.\mathrm{C}_{6} \mathrm{H}_{4}\right), 132.2\left(\mathrm{~s}, \underline{\mathrm{C}}_{\text {meta }}\right.$ of $\left.\mathrm{C}_{6} \mathrm{H}_{4}\right), 134.1\left(\mathrm{~d},{ }^{2} J_{\mathrm{PC}}=3.1, \underline{\mathrm{C}}_{\text {ipso }}\right.$ of $\left.\mathrm{C}_{6} \mathrm{H}_{4}\right) . / \mathbf{~ m} / \mathbf{z}(\mathrm{CI}): 385\left(\mathrm{M}+12 *{ }^{79} \mathrm{Br}, 50\right), 387\left(\mathrm{M}+1{ }^{79} \mathrm{Br}+{ }^{81} \mathrm{Br}, 100\right), 389(\mathrm{M}+12 * 81 \mathrm{Br}, 50), 402(\mathrm{M}+18$ 2*79 $\mathrm{Br}, 38), 404\left(\mathrm{M}+18{ }^{79} \mathrm{Br}+{ }^{81} \mathrm{Br}, 73\right), 406(\mathrm{M}+182 * 81 \mathrm{Br}, 38)$.

Diethyl $\alpha$-iodobenzylphosphonate (7a) $\mathrm{C}_{11} \mathrm{H}_{16} \mathrm{O}_{3} \mathrm{IP}(\mathrm{M}=354):$ 31P-NMR +19.6 (s). / 1 H-NMR 1.08 (t, $\left.{ }^{3} J_{\mathrm{HH}}=7.0,3 \mathrm{H},\left(\mathrm{CH}_{3} \mathrm{CH}_{2} \mathrm{O}\right)_{\mathrm{A}}\right), 1.26\left(\mathrm{t},{ }^{3} \mathrm{~J}_{\mathrm{HH}}=7.0,3 \mathrm{H},\left(\mathrm{CH}_{3} \mathrm{CH}_{2} \mathrm{O}\right)_{\mathrm{B}}\right), 3.76-4.05\left(\mathrm{~m}, 2 \mathrm{H},\left(\mathrm{CH}_{3} \mathrm{CH}_{2} \mathrm{O}\right)_{\mathrm{B}}\right), 4.14$ $\left(\mathrm{p},{ }^{3} J_{\mathrm{HH}}={ }^{3} J_{\mathrm{PH}}=7.0,2 \mathrm{H},\left(\mathrm{CH}_{3} \underline{\mathrm{C}}_{2}\right)_{\mathrm{A}}\right), 4.96\left(\mathrm{~d},{ }^{2} J_{\mathrm{PH}}=13.5,1 \mathrm{H}, \mathrm{C} \underline{\mathrm{HI}}\right), 7.22\left(\mathrm{~m}, 3 \mathrm{H}, \underline{\mathrm{H}}_{\text {meta }}\right.$,para of $\left.\mathrm{C}_{6} \mathrm{H}_{5}\right), 7.48$ $\left(\mathrm{m}, 2 \mathrm{H}, \underline{\mathrm{H}}_{\text {ortho }}\right.$ of $\left.\mathrm{C}_{6} \mathrm{H}_{5}\right) . /{ }^{13} \mathrm{C}-\mathrm{NMR} 15.4\left(\mathrm{~d},{ }^{1} \mathrm{~J}_{\mathrm{PC}}=156.6, \underline{\mathrm{CHI}}\right), 16.5\left(\mathrm{~d},{ }^{3} \mathrm{~J}_{\mathrm{PC}}=6.0,\left(\underline{\mathrm{CH}}_{3} \mathrm{CH}_{2} \mathrm{O}\right)_{\mathrm{A}}\right), 16.7(\mathrm{~d}$, $\left.{ }^{3} J_{\mathrm{PC}}=6.0,\left(\underline{\mathrm{CH}}_{3} \mathrm{CH}_{2} \mathrm{O}\right)_{\mathrm{B}}\right), 64.7\left(\mathrm{~d},{ }^{2} J_{\mathrm{PC}}=7.7,\left(\mathrm{CH}_{3} \underline{\mathrm{CH}}_{2} \mathrm{O}\right)_{\mathrm{B}}\right), 65.0\left(\mathrm{~d},{ }^{2} J_{\mathrm{PC}}=7.3,\left(\mathrm{CH}_{3} \underline{\mathrm{CH}}{ }_{2} \mathrm{O}\right)_{\mathrm{A}}\right), 129.2(\mathrm{~s}$, $\underline{\mathrm{C}}_{\text {meta }}$ of $\left.\mathrm{C}_{6} \mathrm{H}_{5}\right), 129.3$ (s, $\underline{\mathrm{C}}_{\text {para }}$ of $\left.\mathrm{C}_{6} \mathrm{H}_{5}\right), 130.1\left(\mathrm{~d},{ }^{3} J_{\mathrm{PC}}=6.6, \underline{\mathrm{C}}_{\text {ortho }}\right.$ of $\left.\mathrm{C}_{6} \mathrm{H}_{5}\right), 136.5\left(\mathrm{~d},{ }^{2} J_{\mathrm{PC}}=3.0, \underline{\mathrm{C}}_{\text {ipso }}\right.$ of $\left.\mathrm{C}_{6} \mathrm{H}_{5}\right) . / \mathbf{m} / \mathbf{z}(\mathrm{CI}): 355(\mathrm{M}+1,100), 372(\mathrm{M}+18,64)$.

Diethyl $\alpha$-iodo-4-methylbenzylphosphonate (7b) $\mathrm{C}_{12} \mathrm{H}_{18} \mathrm{O}_{3} \mathrm{IP}(\mathrm{M}=368)$ : 31P-NMR +20.7 (s). / 1H-NMR $1.24\left(\mathrm{t},{ }^{3} \mathrm{~J}_{\mathrm{HH}}=7.0,3 \mathrm{H},\left(\mathrm{CH}_{3} \mathrm{CH}_{2} \mathrm{O}\right)_{\mathrm{A}}\right), 1.34\left(\mathrm{t},{ }^{3} \mathrm{~J}_{\mathrm{HH}}=7.0,3 \mathrm{H},\left(\mathrm{CH}_{3} \mathrm{CH}_{2} \mathrm{O}\right)_{\mathrm{B}}\right), 2.37\left(\mathrm{~s}, 3 \mathrm{H}, \mathrm{C}_{6} \mathrm{H}_{4} \mathrm{CH}_{3}\right), 3.73-$ $4.05\left(\mathrm{~m}, 2 \mathrm{H},\left(\mathrm{CH}_{3} \mathrm{C}_{2} \mathrm{O}\right)_{\mathrm{B}}\right), 4.13\left(\mathrm{p},{ }^{3} J_{\mathrm{HH}}={ }^{3} J_{\mathrm{PH}}=7.0,2 \mathrm{H},\left(\mathrm{CH}_{3} \underline{\mathrm{CH}}_{2}\right)_{\mathrm{A}}\right), 5.09\left(\mathrm{~d},{ }^{2} J_{\mathrm{PH}}=13.5,1 \mathrm{H}, \mathrm{C} \underline{\mathrm{HI}}\right), 7.19$ $\left(\mathrm{d},{ }^{3} J_{\mathrm{HH}}=7.2,2 \mathrm{H}, \underline{\mathrm{H}}_{\text {meta }}\right.$ of $\left.\mathrm{C}_{6} \mathrm{H}_{4}\right), 7.41\left(\mathrm{~d},{ }^{3} J_{\mathrm{HH}}=7.2,2 \mathrm{H}, \underline{\mathrm{H}}_{\mathrm{ortho}}\right.$ of $\left.\mathrm{C}_{6} \mathrm{H}_{4}\right) . /{ }^{13} \mathrm{C}-\mathbf{N M R} 16.2\left(\mathrm{~d},{ }^{1} J_{\mathrm{PC}}=160.2\right.$, 
$\underline{\mathrm{CHI}}), 16.6\left(\mathrm{~d},{ }^{3} \mathrm{~J}_{\mathrm{PC}}=6.0, \underline{\mathrm{CH}}_{3} \mathrm{CH}_{2} \mathrm{O}\right), 21.5\left(\mathrm{~s}, \mathrm{C}_{6} \mathrm{H}_{4} \underline{\mathrm{CH}}_{3}\right), 64.2\left(\mathrm{~d},{ }^{2} J_{\mathrm{PC}}=7.3,\left(\mathrm{CH}_{3} \underline{\mathrm{CH}_{2} \mathrm{O}}\right)_{\mathrm{B}}\right), 64.4(\mathrm{~d}$, $\left.{ }^{2} J_{\mathrm{PC}}=6.5,\left(\mathrm{CH}_{3} \underline{\mathrm{CH}}_{2} \mathrm{O}\right)_{\mathrm{A}}\right), 127.5\left(\mathrm{~d},{ }^{3} J_{\mathrm{PC}}=5.8, \underline{\mathrm{C}}_{\text {ortho }}\right.$ of $\left.\mathrm{C}_{6} \mathrm{H}_{4}\right), 129.2\left(\mathrm{~s}, \underline{\mathrm{C}}_{\text {meta }}\right.$ of $\left.\mathrm{C}_{6} \mathrm{H}_{4}\right), 133.6\left(\mathrm{~s}, \underline{\mathrm{C}}_{\mathrm{para}}\right.$ of $\left.\mathrm{C}_{6} \mathrm{H}_{4}\right), 138.1\left(\mathrm{~d},{ }^{2} J_{\mathrm{PC}}=2.8, \underline{\mathrm{C}}_{\mathrm{ipso}}\right.$ of $\left.\mathrm{C}_{6} \mathrm{H}_{4}\right) . / \mathbf{m} / \mathbf{z}(\mathrm{CI}): 369(\mathrm{M}+1,100), 386(\mathrm{M}+18,67)$.

\section{AKNOWLEDGEMENTS}

We thank the Fondation de l'Ecole Polytechnique and the CNRS for financial support to B. I. We are also grateful to Mr. M. Levard (UMR 7652) of the Ecole Polytechnique for the mass spectra.

\section{REFERENCES}

1. Zimmer, H., Bercz, P. J., Maltenieks, O. J., Moore, M. W. J. Am. Chem. Soc. 1965, 87, 2777.

2. Gallagher, M. J., Noerdin, H. Aust. J. Chem.. 1985, 38, 997.

3. Kondo, K., Ohnishi, N., Takemoto, K., Yoshida, H., Yoshida, K. J. Org. Chem.. 1992, 57, 1622.

4. Kumaraswamy, S., Kumara Swamy, K. C. Tetrahedron Lett. 1997, 38, 2183.

5. Hilderbrand, R. L. in The Role of Phosphonates in Living Systems, CRC Press, Boca Raton, FL, 1983.

6. Bergmann, E. D., Shahak, I., Appelbaum, J. Isr. J. Chem. 1968, 6, 73.

7. a) Blackburn, G. M., Kent, D. E. J. Chem. Soc., Chem. Commun. 1981, 511; b) Blackburn, G. M., Kent, D. E. J. Chem. Soc., Perkin. Trans. 1 1986, 913.

8. Gajda, T. Synthesis $1990,717$.

9. Kumaraswamy, S., Senthamizh Selvi, R., Kumara Swamy, K. C. Synthesis 1997, 207.

10. Gajda, T. Phosphorus Sulfur Silicon Relat. Elem. 1990, 53, 327.

11. Green, D., Elgendy, S., Patel, G., Baban, J. A., Skordalakes, E., Husman, W., Kakkar,V. V., Deadman, J. Tetrahedron 1996, 52, 10215 .

12. a) Michaelis, A, Kaehne, R Ber. 1893, 31, 1048; b) Arbuzov, A.E. J. Russ. Phys. Chem. Soc. 1906, $38,687$.

13. (a) Elkik, E., Francesch, C. Bull. Soc. Chim. Fr. 1986, 423; b) Etemad-Moghadam, G., Seyden-Penne, J. Bull. Soc. Chim. Fr. 1985, 448; c) Coutrot, P., Grison, C., Sauvêtre, R. J. Organomet. Chem. 1987, 332, 1; d) Thenappan, A., Burton, D. J. J. Org. Chem. 1990, 55, 4639; e) Gallagher Jr., G., Webb, R. L. Synthesis 1974, 122.

14. Dawson, M. I., Chan, R., Hobbs, P. D., Chao, W., Schiff, L. J. J. Med. Chem. 1983, $26,1282$.

15. Petrova, J., Coutrot, P., Dreux, M., Savignac, P. Synthesis 1975, 658.

16. Grinev, G.V., Chervenyuk, G. I., Dombrovskii, A.V. J. Gen. Chem. USSR (Engl. Trans.), 1969, 39, 1223; Zh. Obshch. Khim.. 1969, 39, 1253.

17. a) Taylor, S. D., Dinaut, A. N., Thadani, A. N., Huang, Z. Tetrahedron Lett. 1996, 37, 8089; b) Taylor, S. D., Kotoris, C. C., Dinaut, A. N., Chen, M-J. Tetrahedron 1998, 54, 1691.

18. Williams, A., Naylor, R. A., Collyer, S. G. J. Chem. Soc., Perkin. II 1973, 25.

19. Gronowitz, S.; Stenhammar, K.; Svensson, L. Heterocycles 1981, 15, 947.

20. Cabares, J.; Mavoungou-Gomes, L. Bull. Soc. Chim. Fr. 1986, 401.

21. Lee, S.-G.; Bentrude, W. G. Phosphorus Sulfur 1988, 35, 219.

22. Schwender, C. F.; Beers, S. A.; Malloy, E. A.; Cinicola, J. J. Bioorg. Med. Chem. Lett. 1996, 6, 311.

23. Mu, Y. Q.; Gibbs, R. A. Bioorg. Med. Chem. 1997, 5, 1327.

24. Lugovkin, B. P., Arbuzov, B. A. Dokl. Akad. Nauk SSSR 1948, 59, 1301; Chem. Abstr. 1948, 42, 7265.

25. Raju, B.; Okun, I.; Stavros, F.; Chan, M. F. Bioorg. Med. Chem. Lett. 1997, 7, 939.

26. Nasser, J.; About-Jaudet, E.; Collignon, N. Phosphorus Sulfur Silicon Relat. Elem. 1990, 54, 171.

27. Savignac, P.; Lavielle, G. Bull. Soc. Chim. Fr. 1974, 1506. 
28. Kennedy, G.; Perboni, A. D. Tetrahedron Lett. 1996, 37, 7611.

29. Franke, A., Frickel, F-F., Schlecker, R., Thieme, P. C. Synthesis 1979, 712.

30. Taylor, W. P.; Zhang, Z.-Y.; Widlanski, T. S. Bioorg. Med.Chem. 1996, 4, 1515.

31. Tsai, H.-J. Tetrahedron Lett. 1996, 37, 629. 